\title{
ADIC FINITENESS: BOUNDING HOMOLOGY AND APPLICATIONS
}

\author{
SEAN SATHER-WAGSTAFF AND RICHARD WICKLEIN
}

\begin{abstract}
We prove a versions of amplitude inequalities of Iversen, Foxby and Iyengar, and Frankild and Sather-Wagstaff that replace finite generation conditions with adic finiteness conditions. As an application, we prove that a local ring $R$ of prime characteristic is regular if and only if for some proper ideal $\mathfrak{b}$ the derived local cohomology complex $\mathbf{R} \Gamma_{\mathfrak{b}}(R)$ has finite flat dimension when viewed through some positive power of the Frobenius endomorphism.
\end{abstract}

\section{Contents}

1. Introduction

2. Background

3. Bounding Homology I: Maximal Support

4. Bounding Homology II: Over a Ring Homomorphism

5. Bounding Homology III: Modules

6. Applications

Acknowledgments

References

\section{INTRODUCTION}

Throughout this paper let $R$ and $S$ be commutative noetherian rings, let $\mathfrak{a} \subsetneq R$ be a proper ideal of $R$, and let $\widehat{R}^{\mathfrak{a}}$ be the $\mathfrak{a}$-adic completion of $R$. Let $K=K^{R}(\underline{x})$ denote the Koszul complex over $R$ on a generating sequence $\underline{x}=x_{1}, \ldots, x_{n}$ for a. We work in the derived category $\mathcal{D}(R)$ with objects the $R$-complexes indexed homologically $X=\cdots \rightarrow X_{i} \rightarrow X_{i-1} \rightarrow \cdots$. The $i$ th shift (or suspension) of $X$ is denoted $\Sigma^{i} X$. We consider the following full triangulated subcategories of $\mathcal{D}(R)$.

$\mathcal{D}_{\mathrm{b}}(R)$ : objects are the complexes $X$ with $\mathrm{H}_{i}(X)=0$ for $|i| \gg 0$.

$\mathcal{D}^{\mathrm{f}}(R)$ : objects are the complexes $X$ with $\mathrm{H}_{i}(X)$ finitely generated for all $i$.

$\mathcal{D}_{\mathrm{b}}^{\mathrm{f}}(R):=\mathcal{D}^{\mathrm{f}}(R) \cap \mathcal{D}_{\mathrm{b}}(R)$.

Isomorphisms in $\mathcal{D}(R)$ are identified by the symbol $\simeq$. The appropriately derived functors of $\operatorname{Hom}_{R}(, R)$ and $\otimes_{R}$ are $\mathbf{R} \operatorname{Hom}_{R}$ and $\otimes_{R}^{\mathbf{L}}$. See Section 2 for background material and, e.g., $12,31,32$ for foundations.

2010 Mathematics Subject Classification. 13C12, 13D05, 13D07, 13D09 .

Key words and phrases. Adic finiteness; amplitude inequality, co-support, flat dimension, Frobenius endomorphism, injective dimension, projective dimension, support.

Sean Sather-Wagstaff was supported in part by a grant from the NSA. 
This work is part 3 of a series of papers exploring notions of support and finiteness of $R$-complexes. It builds on our previous papers [27, 29], and it is used in the papers [25, 26, 28]. It is heavily influenced by Foxby and Iyengar's paper [10] and the non-local extension in 11 .

A major point of 10 is to prove an amplitude inequality extending a result of Iversen 15 to the realm of unbounded complexes. This implies that, given a local ring homomorphism $R \rightarrow S$ with complexes $X \in \mathcal{D}^{\mathrm{f}}(R)$ and $F \in \mathcal{D}_{\mathrm{b}}^{\mathrm{f}}(S)$ such that $F \not 0$ and $\operatorname{fd}_{R}(F)<\infty$, then one has $X \in \mathcal{D}_{\mathrm{b}}(R)$ if and only if $F \otimes \otimes_{R}^{\mathbf{L}} X \in \mathcal{D}_{\mathrm{b}}(R)$; that is, one has $\mathrm{H}_{i}(X)=0$ for $|i| \gg 0$ if and only if $\operatorname{Tor}_{i}^{R}(F, X)=0$ for $|i| \gg 0$. This is extended to the non-local arena in [1].

In the current paper, we extend these and other results to the realm of complexes that do not necessarily have finitely generated homology modules, but instead have finitely generated Koszul homology modules and restricted support. These are the "adically finite complexes", introduced in [29]. For instance, an $R$-module $M$ is $\mathfrak{a}$-adically finite if it is a-torsion and has $\mathrm{H}_{i}\left(K \otimes_{R} M\right)$ finitely generated for all $i$. See Definition 2.4 for the general definition. In this context, our generalization of the results from the previous paragraph is the following, which is a consequence of Theorem 4.1 below.

Theorem 1.1. Let $\varphi: R \rightarrow S$ be a ring homomorphism such that $\mathfrak{a} S \neq S$, and let $\varphi^{*}$ be the induced map $\operatorname{Spec}(S) \rightarrow \operatorname{Spec}(R)$. Let $F \in \mathcal{D}_{b}(S)$ be a $S$-adically finite such that $\mathrm{fd}_{R}(F)<\infty$ and $\varphi^{*}\left(\operatorname{supp}_{S}(F)\right) \supseteq \mathrm{V}(\mathfrak{a}) \bigcap \mathrm{m}-\operatorname{Spec}(R)$. Let $X \in \mathcal{D}(R)$ be such that $\operatorname{supp}_{R}(X) \subseteq \mathrm{V}(\mathfrak{a})$ and $K \otimes_{R}^{\mathbf{L}} X \in \mathcal{D}^{f}(R)$. Then one has $X \in \mathcal{D}_{b}(R)$ if and only if $F \otimes_{R}^{\mathbf{L}} X \in \mathcal{D}_{b}(R)$.

The point of this and most of the other results of this paper is that, in the presence of reasonable support conditions, one can relax homologically finite assumptions to adically finite assumptions. Sections 3 [5 contains numerous results akin to Theorem 1.1 with various derived functors and finiteness conditions. It should be reiterated that these results are all applied in our subsequent work, especially in 25]. We also note that many of the results of Section 3 are new even when the adically finite condition is replaced with the more restrictive assumption of being in $\mathcal{D}_{\mathrm{b}}^{\mathrm{f}}(R)$. For instance, the next result is a special case of Theorem 3.15 .

Theorem 1.2. Let $F \in \mathcal{D}_{b}^{f}(R)$ be such that $\operatorname{fd}_{R}(F)<\infty$. Let $Z \in \mathcal{D}(R)$ be such that $\operatorname{supp}_{R}(Z) \subseteq \operatorname{supp}_{R}(F)$. One has $Z \in \mathcal{D}_{b}(R)$ if and only if $F \otimes \mathbb{R}_{R}^{\mathbf{L}} Z \in \mathcal{D}_{b}(R)$.

Section [6] applies these results to the study of homological properties of local ring homomorphisms. For instance, the next result, contained in Theorem 6.2 is a significant extension of [10, Theorem 3.3].

Theorem 1.3. Let $(R, \mathfrak{m})$ be a local ring of prime characteristic, and let $\varphi: R \rightarrow R$ be the Frobenius endomorphism. For an $R$-complex $X$, let $\varphi^{t} X$ denote the complex $X$ viewed as an $R$-complex by restriction of scalars along the $n$-fold composition $\varphi^{t}$. Then the following conditions are equivalent.

(i) $R$ is regular.

(ii) $\varphi^{t}$ is flat for each integer $t \geqslant 1$.

(iii) One has $\operatorname{fd}_{R}\left(\varphi^{t} \mathbf{R} \Gamma_{\mathfrak{b}}(R)\right)<\infty$ for some integer $t \geqslant 1$ and some ideal $\mathfrak{b} \subseteq \mathfrak{m}$.

(iv) One has $\operatorname{fd}_{R}\left(\varphi^{t} F\right)<\infty$ for some integer $t \geqslant 1$ for some $\mathfrak{b}$-adically finite $R$-complex $F \not 40$ with $\mathrm{fd}_{R}(F)<\infty$ for some ideal $\mathfrak{b} \subseteq \mathfrak{m}$. 
Here $\mathbf{R} \Gamma_{\mathfrak{b}}(R)$ is the derived local cohomology complex with respect to $\mathfrak{b}$. Further applications are contained in the subsequent [25].

It is worth noting that there are a number of substitutes for homological finiteness (e.g., for finite generation of modules) in the literature. For instance, Simon 30] considers (complexes of) a-adically complete modules. We observe that if $X \in \mathcal{D}(R)$ is such that co-supp $\operatorname{su}_{R}(X) \subseteq \mathrm{V}(\mathfrak{a})$, then $X$ is isomorphic in $\mathcal{D}(R)$ to a complex of flat complete $R$-modules, namely, the complex $\Lambda^{\mathfrak{a}}(F) \simeq \mathbf{L} \Lambda^{\mathfrak{a}}(X) \simeq X$ where $F \simeq X$ is a semi-flat resolution; see Fact 2.2. However, our a-adically finite complexes are different from this. Indeed, let $(R, \mathfrak{m}, k)$ be a local ring of positive depth. The injective hull $E:=E_{R}(k)$ is not $\mathfrak{m}$-adically separated, so it is not isomorphic in $\mathcal{D}(R)$ to a complex of complete $R$-modules. On the other hand, $E$ is $\mathfrak{m}$-adically finite by [29, Proposition 7.8(b)].

Another replacement for homological finiteness is Porta, Shaul, and Yekutieli's notion of cohomological cofiniteness, from [24. The difference between this notion and ours is discussed in depth in [28, Section 6].

\section{BACKGROUND}

Derived Categories. We expand our menagerie of categories from the introduction to include the next full triangulated subcategories of $\mathcal{D}(R)$.

$\mathcal{D}_{+}(R)$ : objects are the complexes $X$ with $\mathrm{H}_{i}(X)=0$ for $i \ll 0$.

$\mathcal{D}_{-}(R)$ : objects are the complexes $X$ with $\mathrm{H}_{i}(X)=0$ for $i \gg 0$.

Doubly ornamented subcategories are intersections, e.g., $\mathcal{D}_{+}^{\mathrm{f}}(R):=\mathcal{D}^{\mathrm{f}}(R) \bigcap \mathcal{D}_{+}(R)$.

Homological Dimensions. An $R$-complex $F$ is semi-flat ${ }^{1}$ if it consists of flat $R$ modules and the functor $F \otimes_{R}$ - respects quasiisomorphisms, that is, if it respects injective quasiisomorphisms (see [2, 1.2.F]). A semi-flat resolution of an $R$-complex $X$ is a quasiisomorphism $F \stackrel{\simeq}{\rightarrow} X$ such that $F$ is semi-flat. An $R$-complex $X$ has finite flat dimension if it has a bounded semi-flat resolution; specifically, we have

$$
\mathrm{fd}_{R}(X)=\inf \left\{\sup \left\{i \mid F_{i} \neq 0\right\} \mid F \stackrel{\simeq}{\rightarrow} X \text { is a semi-flat resolution }\right\} .
$$

The projective and injective versions of these notions are defined similarly.

For the following items, consult [2, Section 1] or [3, Chapters 3 and 5]. Bounded below complexes of flat modules are semi-flat, bounded below complexes of projective modules are semi-projective, and bounded above complexes of injective modules are semi-injective. Semi-projective $R$-complexes are semi-flat. Every $R$-complex admits a semi-projective (hence, semi-flat) resolution and a semi-injective resolution.

Derived Local (Co)homology. The next notions go back to Grothendieck [13] and Matlis 20, 21; see also 1, 19]. Let $\Lambda^{\mathfrak{a}}$ denote the a-adic completion functor, and let $\Gamma_{\mathfrak{a}}$ be the $\mathfrak{a}$-torsion functor, i.e., for an $R$-module $M$ we have

$$
\Lambda^{\mathfrak{a}}(M)=\widehat{M}^{\mathfrak{a}} \quad \Gamma_{\mathfrak{a}}(M)=\left\{x \in M \mid \mathfrak{a}^{n} x=0 \text { for } n \gg 0\right\} .
$$

A module $M$ is $\mathfrak{a}$-torsion if $\Gamma_{\mathfrak{a}}(M)=M$.

The associated left and right derived functors (i.e., derived local homology and cohomology functors) are $\mathbf{L} \Lambda^{\mathfrak{a}}(-)$ and $\mathbf{R} \Gamma_{\mathfrak{a}}(-)$. Specifically, given an $R$-complex

\footnotetext{
${ }^{1}$ In the literature, semi-flat complexes are sometimes called "K-flat" or "DG-flat".
} 
$X \in \mathcal{D}(R)$ and a semi-flat resolution $F \stackrel{\simeq}{\rightarrow} X$ and a semi-injective resolution $X \stackrel{\simeq}{\rightarrow} I$, then we have $\mathbf{L} \Lambda^{\mathfrak{a}}(X) \simeq \Lambda^{\mathfrak{a}}(F)$ and $\mathbf{R} \Gamma_{\mathfrak{a}}(X) \simeq \Gamma_{\mathfrak{a}}(I)$.

Support and Co-support. The following notions are due to Foxby [9] and Benson, Iyengar, and Krause [5].

Definition 2.1. Let $X \in \mathcal{D}(R)$. The small and large support and small co-support of $X$ are

$$
\begin{aligned}
\operatorname{supp}_{R}(X) & =\left\{\mathfrak{p} \in \operatorname{Spec}(R) \mid \kappa(\mathfrak{p}) \otimes_{R}^{\mathbf{L}} X \not 0\right\} \\
\operatorname{Supp}_{R}(X) & =\left\{\mathfrak{p} \in \operatorname{Spec}(R) \mid R_{\mathfrak{p}} \otimes_{R}^{\mathbf{L}} X \neq 0\right\} \\
\operatorname{co} \operatorname{supp}_{R}(X) & =\left\{\mathfrak{p} \in \operatorname{Spec}(R) \mid \mathbf{R H o m}_{R}(\kappa(\mathfrak{p}), X) \neq 0\right\}
\end{aligned}
$$

where $\kappa(\mathfrak{p}):=R_{\mathfrak{p}} / \mathfrak{p} R_{\mathfrak{p}}$. We have a notion of Co-supp $\sup _{R}(X)$, as well, but do not need it here.

Much of the following is from [9] when $X$ and $Y$ are appropriately bounded and from [4, 5] in general. We refer to [29] as a matter of convenience.

Fact 2.2. Let $X, Y \in \mathcal{D}(R)$. Then we have $\operatorname{supp}_{R}(X)=\emptyset$ if and only if $X \simeq 0$ if and only if co-supp $(X)=\emptyset$, because of [29, Fact 3.4 and Proposition 4.7(a)]. Also, by [29, Propositions 3.12 and 4.10] we have

$$
\begin{aligned}
\operatorname{supp}_{R}\left(X \otimes \otimes_{R}^{\mathbf{L}} Y\right) & =\operatorname{supp}_{R}(X) \bigcap \operatorname{supp}_{R}(Y) \\
\operatorname{co-supp}_{R}\left(\mathbf{R H o m}_{R}(X, Y)\right) & =\operatorname{supp}_{R}(X) \bigcap \operatorname{co-supp}_{R}(Y) .
\end{aligned}
$$

In addition, we know that $\operatorname{supp}_{R}(X) \subseteq \mathrm{V}(\mathfrak{a})$ if and only if $X \simeq \mathbf{R} \Gamma_{\mathfrak{a}}(X)$ if and only if each homology module $\mathrm{H}_{i}(X)$ is $\mathfrak{a}$-torsion, by [29, Proposition 5.4] and [23, Corollary 4.32]. Similarly, we have co-supp ${ }_{R}(X) \subseteq \mathrm{V}(\mathfrak{a})$ if and only if $\mathbf{L} \Lambda^{\mathfrak{a}}(X) \simeq X$, by [29, Propositions 5.9].

Adic Finiteness. The next fact and definition from [29] take their cues from work of Hartshorne [14, Kawasaki [16, 17, and Melkersson [22].

Fact 2.3 ([29, Theorem 1.3]). For $X \in \mathcal{D}_{\mathrm{b}}(R)$, the next conditions are equivalent.

(i) One has $K^{R}(\underline{y}) \otimes_{R}^{\mathrm{L}} X \in \mathcal{D}_{\mathrm{b}}^{\mathrm{f}}(R)$ for some (equivalently for every) finite generating sequence $\underline{y}$ of $\mathfrak{a}$.

(ii) One has $X \otimes_{R}^{\mathrm{L}} R / \mathfrak{a} \in \mathcal{D}^{\mathrm{f}}(R)$.

(iii) One has $\operatorname{RHom}_{R}(R / \mathfrak{a}, X) \in \mathcal{D}^{\mathrm{f}}(R)$.

Definition 2.4. An $R$-complex $X \in \mathcal{D}_{\mathrm{b}}(R)$ is a-adically finite if it satisfies the equivalent conditions of Fact 2.3 and $\operatorname{supp}_{R}(X) \subseteq \mathrm{V}(\mathfrak{a})$.

Example 2.5. Let $X \in \mathcal{D}_{\mathrm{b}}(R)$ be given.

(a) If $X \in \mathcal{D}_{\mathrm{b}}^{\mathrm{f}}(R)$, then we have $\operatorname{supp}_{R}(X)=\mathrm{V}(\mathfrak{b})$ for some ideal $\mathfrak{b}$, and it follows that $X$ is $\mathfrak{a}$-adically finite whenever $\mathfrak{a} \subseteq \mathfrak{b}$. (The case $\mathfrak{a}=0$ is from 29, Proposition 7.8], and the general case follows readily.)

(b) $K$ and $\mathbf{R} \Gamma_{\mathfrak{a}}(R)$ are $\mathfrak{a}$-adically finite, by [29, Fact 3.4 and Theorem 7.10].

(c) The homology modules of $X$ are artinian if and only if there is an ideal $\mathfrak{a}$ of finite colength (i.e., such that $R / \mathfrak{a}$ is artinian) such that $X$ is a-adically finite, by [27, Proposition 5.11]. 
Fact 2.6. Let $X \in \mathcal{D}(R)$ be given. It is straightforward to show that one has $\operatorname{supp}_{R}(X) \subseteq \operatorname{Supp}_{R}(X)$. A little more work allows one to show that $\operatorname{supp}_{R}(X) \subseteq$ $\mathrm{V}(\mathfrak{a})$ if and only if $\operatorname{Supp}_{R}(X) \subseteq \mathrm{V}(\mathfrak{a})$; see 29, Proposition 3.15]. If $X$ is a-adically finite, then one has more, by [29, Proposition 7.11]: the set $\operatorname{supp}_{R}(X)=\operatorname{Supp}_{R}(X)$ is Zariski-closed in $\operatorname{Spec}(R)$.

Bookkeeping. We use some convenient accounting tools, due in this generality to Foxby and Iyengar [8, 10.

Definition 2.7. The supremum, infimum, amplitude, $\mathfrak{a}$-depth, and $\mathfrak{a}$-width of an $R$-complex $Z$ are

$$
\begin{aligned}
\sup (Z) & =\sup \left\{i \in \mathbb{Z} \mid \mathrm{H}_{i}(Z) \neq 0\right\} \\
\inf (Z) & =\inf \left\{i \in \mathbb{Z} \mid \mathrm{H}_{i}(Z) \neq 0\right\} \\
\operatorname{amp}(Z) & =\sup (Z)-\inf (Z) \\
\operatorname{depth}_{\mathfrak{a}}(Z) & =-\sup (\mathbf{R H o m} \\
\operatorname{width}_{\mathfrak{a}}(Z) & =\inf \left((R / \mathfrak{a}) \otimes_{R}^{\mathbf{L}} Z\right)
\end{aligned}
$$

with the conventions $\sup \emptyset=-\infty$ and $\inf \emptyset=\infty$. When $(R, \mathfrak{m})$ is local, one sets $\operatorname{depth}_{R}(Z):=\operatorname{depth}_{\mathfrak{m}}(Z)$ and $\operatorname{width}_{R}(Z):=\operatorname{width}_{\mathfrak{m}}(Z)$.

In the following fact, items ( (a) $-($ b are by definition, and item (IC) is from 2 , Theorems 2.4.F and P] and [8, Lemma 2.1].

Fact 2.8. Let $Y, Z \in \mathcal{D}(R)$.

(a) One has $\sup (Z)<\infty$ if and only if $Z \in \mathcal{D}_{-}(R)$. Also, one has $\inf (Z)>-\infty$ if and only if $Z \in \mathcal{D}_{+}(R)$, and one has $\operatorname{amp}(Z)<\infty$ if and only if $Z \in \mathcal{D}_{\mathrm{b}}(R)$.

(b) The following conditions are equivalent:

(i) $Z \simeq 0$, (ii) $\sup (Z)=-\infty$, (iii) $\inf (Z)=\infty$, and (iv) $\operatorname{amp}(Z)=-\infty$.

(c) There are inequalities

$$
\begin{gathered}
\inf (Y)+\inf (Z) \leqslant \inf \left(Y \otimes_{R}^{\mathbf{L}} Z\right) \\
\sup \left(Y \otimes_{R}^{\mathbf{L}} Z\right) \leqslant \sup (Y)+\operatorname{fd}_{R}(Z) \\
\inf (Z)-\operatorname{pd}_{R}(Y) \leqslant \inf (\mathbf{R H o m} R(Y, Z)) \\
\sup \left(\mathbf{R H o m}_{R}(Y, Z)\right) \leqslant \sup (Z)-\inf (Y)
\end{gathered}
$$

Koszul Complexes. We refer to parts of the following as the "self-dual nature" of the Koszul complex.

Fact 2.9. Let $\mathbf{y}=y_{1}, \ldots, y_{m} \in R$, and consider the Koszul complex $L:=K^{R}(\mathbf{y})$. Then we have isomorphisms in $\mathcal{D}(R)$

$$
\begin{gathered}
L \simeq \Sigma^{n} \mathbf{R H o m}_{R}(L, R) \\
L \otimes_{R}^{\mathbf{L}} X \simeq \Sigma^{n} \mathbf{R} \operatorname{Hom}_{R}(L, R) \otimes_{R}^{\mathbf{L}} X \simeq \Sigma^{n} \mathbf{R} \operatorname{Hom}_{R}(L, X) \\
\mathbf{R H o m}_{R}\left(\mathbf{R} \operatorname{Hom}_{R}(L, X), Y\right) \simeq L \otimes_{R}^{\mathbf{L}} \mathbf{R H o m}(X, Y) \simeq \mathbf{R H o m}_{R}\left(X, L \otimes_{R}^{\mathbf{L}} Y\right) .
\end{gathered}
$$

See, e.g., [27, Remark 2.2].

The following facts are from [27, Lemmas 3.1-3.2].

Fact 2.10. Let $Z \in \mathcal{D}(R)$, let $\mathbf{y}=y_{1}, \ldots, y_{m} \in \mathfrak{a}$, and set $L:=K^{R}(\mathbf{y})$ and $\mathfrak{b}=(\mathbf{y}) R$. Assume that $\operatorname{supp}_{R}(Z) \subseteq \mathrm{V}(\mathfrak{a})$, e.g., that each homology module $\mathrm{H}_{i}(Z)$ is annihilated by a power of $\mathfrak{a}$. 
(a) There are (in)equalities

$$
\begin{aligned}
\inf \left(L \otimes_{R}^{\mathbf{L}} Z\right) & \leqslant m+\inf (Z) & \sup \left(L \otimes_{R}^{\mathbf{L}} Z\right) & =m+\sup (Z) \\
\operatorname{amp}(Z) & \leqslant \operatorname{amp}\left(L \otimes_{R}^{\mathbf{L}} Z\right) & \operatorname{depth}_{\mathfrak{b}}(Z) & =-\sup (Z) .
\end{aligned}
$$

(b) For each $* \in\{+,-, \mathrm{b}\}$, one has $L \otimes_{R}^{\mathbf{L}} Z \in \mathcal{D}_{*}(R)$ if and only if $Z \in \mathcal{D}_{*}(R)$.

Note that some items in the next result use $L$, while others use $K$.

Fact 2.11. Let $Z \in \mathcal{D}(R)$, let $\mathbf{y}=y_{1}, \ldots, y_{m} \in \mathfrak{a}$, and set $L:=K^{R}(\mathbf{y})$ and

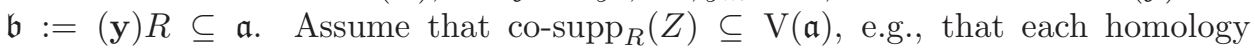
module $\mathrm{H}_{i}(Z)$ is annihilated by a power of $\mathfrak{a}$.

(a) There are (in)equalities

$$
\begin{gathered}
\operatorname{width}_{\mathfrak{b}}(Z)=\inf (Z)=\inf \left(L \otimes_{R}^{\mathbf{L}} Z\right) \\
\sup (Z)-n \leqslant \sup \left(K \otimes_{R}^{\mathbf{L}} Z\right) \\
\operatorname{amp}(Z)-n \leqslant \operatorname{amp}\left(K \otimes_{R}^{\mathbf{L}} Z\right) .
\end{gathered}
$$

(b) For each $* \in\{+,-, \mathrm{b}\}$, one has $K \otimes_{R}^{\mathrm{L}} Z \in \mathcal{D}_{*}(R)$ if and only if $Z \in \mathcal{D}_{*}(R)$.

\section{Bounding Homology I: Maximal Support}

In this section, we extend results from [8, 10, 11 to the adically finite arena.

Bounding by Ext. We begin with two improvements of a result from 8 to the adically finite realm. Note that each of these results assumes enough boundedness on $X$ and $Y$ to imply that $\mathbf{R H o m}_{R}(X, Y) \in \mathcal{D}_{-}(R)$, in contrast with other results below. The point of our results is to give specific ranges for non-vanishing homology. For instance, Proposition 3.1, in conjunction with Fact 2.8( (c)), implies that $\mathrm{H}_{i}\left(\mathbf{R} \operatorname{Hom}_{R}(X, Y)\right) \neq 0$ for some $i \in\{\sup (Y)-\sup (X), \ldots, \sup (Y)-\inf (X)\}$, when $Y \not 00$ Also, as far as we know, the non-trivial implication in part Proposition 3.1 (b) does not follow just from support considerations; and similarly for other results below.

Proposition 3.1. Let $X \in \mathcal{D}_{b}(R)$ be a-adically finite, and let $Y \in \mathcal{D}_{-}(R)$ be such that $\operatorname{supp}_{R}(Y) \subseteq \mathrm{V}(\mathfrak{a})=\operatorname{supp}_{R}(X)$.

(a) Then there is an inequality

$$
\sup (Y)-\sup (X)-n \leqslant \sup (\mathbf{R H o m} R(X, Y)) .
$$

(b) One has $Y \simeq 0$ if and only if $\mathbf{R H o m}_{R}(X, Y) \simeq 0$.

Proof. (回) Claim: If $X \in \mathcal{D}_{\mathrm{b}}^{\mathrm{f}}(R)$, then $\sup (Y)-\sup (X) \leqslant \sup \left(\mathbf{R H o m}_{R}(X, Y)\right)$. Indeed, the condition $\operatorname{supp}_{R}(Y) \subseteq \mathrm{V}(\mathfrak{a})$ implies that $\mathrm{H}_{i}(Y)$ is $\mathfrak{a}$-torsion for all $i$, so

$$
\operatorname{Supp}_{R}\left(\mathrm{H}_{i}(Y)\right) \subseteq \mathrm{V}(\mathfrak{a})=\operatorname{supp}_{R}(X)=\operatorname{Supp}_{R}(X)
$$

by Fact 2.6. Thus, the desired inequality is from [8, Proposition 2.2] 3

\footnotetext{
${ }^{2}$ The conditions $Y \in \mathcal{D}_{-}(R)$ and $Y \not \chi 0$ imply that $\sup (Y) \in \mathbb{Z}$. Note that we do not need to make a similar assumption for $X$, since the condition $\mathfrak{a} \neq R$ implies that $\operatorname{supp}_{R}(X)=\mathrm{V}(\mathfrak{a}) \neq 0$, so we have $X \not \varkappa 0$, and thus $\sup (X), \inf (X) \in \mathbb{Z}$.

${ }^{3}$ Note that complexes in [8] are indexed cohomologically, so one has to translate [8] Proposition 2.2] carefully.
} 
Now we prove the inequality $\sup (Y)-\sup (X)-n \leqslant \sup \left(\mathbf{R H o m}_{R}(X, Y)\right)$ in general. By assumption, we have $K \otimes_{R}^{\mathbf{L}} X \in \mathcal{D}_{\mathrm{b}}^{\mathrm{f}}(R)$, so the above claim explains the second step in the next display.

$$
\begin{aligned}
\sup (Y)-\sup (X)-n & =\sup (Y)-\sup \left(K \otimes_{R}^{\mathbf{L}} X\right) \\
& \leqslant \sup \left(\mathbf{R} \operatorname{Hom}_{R}\left(K \otimes_{R}^{\mathbf{L}} X, Y\right)\right) \\
& =\sup \left(\mathbf{R} \operatorname{Hom}_{R}\left(K, \mathbf{R} \operatorname{Hom}_{R}(X, Y)\right)\right) \\
& \leqslant \sup \left(\mathbf{R} \operatorname{Hom}_{R}(X, Y)\right)-\inf K \\
& =\sup \left(\mathbf{R} \operatorname{Hom}_{R}(X, Y)\right)
\end{aligned}
$$

The first step is from Fact 2.10(国) because $\operatorname{supp}_{R}(X) \subseteq \mathrm{V}(\mathfrak{a})$. The third step is by adjointness $\mathbf{R} \operatorname{Hom}_{R}\left(K \otimes \mathbb{R}_{R}^{\mathbf{L}} X, Y\right) \simeq \mathbf{R} \operatorname{Hom}_{R}\left(K, \mathbf{R} \operatorname{Hom}_{R}(X, Y)\right)$, and the fourth step is from Fact 2.8 (c). The last step is due to the equality $\inf (K)=0$.

(b) The forward implication is standard. For the converse, assume that we have $\mathbf{R H o m}_{R}(X, Y) \simeq 0$. It follows that

$$
\sup (Y)-\sup (X)-n \leqslant \sup \left(\mathbf{R} \operatorname{Hom}_{R}(X, Y)\right)=-\infty .
$$

Our assumptions guarantee that $\sup (X) \in \mathbb{Z}$, so we conclude that $\sup (Y)=-\infty$, thus $Y \simeq 0$.

Proposition 3.2. Let $X \in \mathcal{D}_{b}(R)$ be a-adically finite, and let $Y \in \mathcal{D}_{-}(R)$ be such that co-supp $\sup _{R}(Y) \subseteq \mathrm{V}(\mathfrak{a})=\operatorname{supp}_{R}(X)$. Then there is an inequality

$$
\sup (Y)-\sup (X)-3 n \leqslant \sup \left(\mathbf{R H o m}_{R}(X, Y)\right) .
$$

Proof. The desired inequality follows from the next sequence.

$$
\begin{aligned}
\sup \left(\mathbf{R H o m}_{R}(X, Y)\right) & \geqslant \sup \left(K \otimes_{R}^{\mathbf{L}} \mathbf{R} \operatorname{Hom}_{R}(X, Y)\right)-n \\
& \geqslant \sup \left(\mathbf{R} \operatorname{Hom}_{R}\left(X, K \otimes_{R}^{\mathbf{L}} Y\right)\right)-n \\
& \geqslant \sup \left(K \otimes_{R}^{\mathbf{L}} Y\right)-\sup (X)-2 n \\
& \geqslant \sup (Y)-\sup (X)-3 n
\end{aligned}
$$

The first inequality is from Fact 2.8( (c) , and the second inequality is from tensorevaluation 2.9 . Fact 2.2 implies that $\operatorname{supp}_{R}\left(K \otimes_{R}^{\mathrm{L}} Y\right) \subseteq \operatorname{supp}_{R}(K) \subseteq \mathrm{V}(\mathfrak{a})$, so the third inequality is from Proposition 3.1(国). The fourth inequality is from Fact 2.11(a).

Our next results are more along the lines of Theorem 1.1 from the introduction: in the presence of support and (adic) finiteness assumptions, boundedness or triviality of $\mathbf{R H o m}_{R}(P, Y)$ implies the same for $Y$. See Lemma 3.10 for an improvement on the following.

Lemma 3.3. Let $P \in \mathcal{D}_{b}^{f}(R)$ be such that $P \nsucceq 0$ and $\operatorname{pd}_{R}(P)<\infty$. Let $Y \in \mathcal{D}(R)$ be such that $\operatorname{supp}_{R}(Y) \subseteq \operatorname{supp}_{R}(P)$.

(a) One has $\sup (Y)-\sup (P) \leqslant \sup \left(\mathbf{R H o m}_{R}(P, Y)\right)$.

(b) One has $Y \in \mathcal{D}_{-}(R)$ if and only if $\mathbf{R H o m}_{R}(P, Y) \in \mathcal{D}_{-}(R)$.

(c) One has $Y \simeq 0$ if and only if $\mathbf{R H o m}_{R}(P, Y) \simeq 0$.

Proof. Note first that the conditions $0 \neq P \in \mathcal{D}_{\mathrm{b}}^{\mathrm{f}}(R)$ imply that $\operatorname{supp}_{R}(P)=\mathrm{V}(\mathfrak{a})$ for some $\mathfrak{a} \neq R$. 
(回) Because of the assumption $\operatorname{supp}_{R}(Y) \subseteq \mathrm{V}(\mathfrak{a})$, the first step in the next sequence is from Fact 2.10(a).

$$
\begin{aligned}
-\sup (Y) & =\operatorname{depth}_{\mathfrak{a}}(Y) \\
& =\inf \left\{\operatorname{depth}_{R_{\mathfrak{p}}}\left(Y_{\mathfrak{p}}\right) \mid \mathfrak{p} \in \mathrm{V}(\mathfrak{a})\right\} \\
& =\inf \left\{\operatorname{depth}_{R_{\mathfrak{p}}}\left(\mathbf{R H o m}_{R_{\mathfrak{p}}}\left(P_{\mathfrak{p}}, Y_{\mathfrak{p}}\right)\right)-\operatorname{width}_{R_{\mathfrak{p}}}\left(P_{\mathfrak{p}}\right) \mid \mathfrak{p} \in \mathrm{V}(\mathfrak{a})\right\} \\
& =\inf \left\{\operatorname{depth}_{R_{\mathfrak{p}}}\left(\mathbf{R H o m} \operatorname{Hom}_{R}(P, Y)_{\mathfrak{p}}\right)-\inf \left(P_{\mathfrak{p}}\right) \mid \mathfrak{p} \in \mathrm{V}(\mathfrak{a})\right\} \\
& \geqslant-\sup (\mathbf{R H o m} \\
R & (P, Y))-\sup (P) .
\end{aligned}
$$

The second and third steps are from [10, Propositions 2.10 and 4.6]; note that the third step uses the assumption $\operatorname{supp}_{R}(P)=\mathrm{V}(\mathfrak{a})$. For the fourth step, we use the conditions $P \in \mathcal{D}_{\mathrm{b}}^{\mathrm{f}}(R)$ and $\operatorname{pd}_{R}(P)<\infty$ to conclude that $\mathbf{R} \operatorname{Hom}_{R_{\mathfrak{p}}}\left(P_{\mathfrak{p}}, Y_{\mathfrak{p}}\right) \simeq$ $\mathbf{R H o m}_{R}(P, Y)_{\mathfrak{p}}$, e.g., by [7, Proposition 2.1(iii)]; for the equality width $R_{\mathfrak{p}}\left(P_{\mathfrak{p}}\right)=$ $\inf \left(P_{\mathfrak{p}}\right)$, see, e.g., 6, (1.2.1)]. To explain the last step in this display, we have the following, by definition and Fact $2.8($ (C)

$$
\begin{aligned}
\operatorname{depth}_{R_{\mathfrak{p}}}\left(\mathbf{R} \operatorname{Hom}_{R}(P, Y)_{\mathfrak{p}}\right) & =-\sup \left(\mathbf{R} \operatorname{Hom}_{R_{\mathfrak{p}}}\left(\kappa(\mathfrak{p}), \mathbf{R} \operatorname{Hom}_{R}(P, Y)_{\mathfrak{p}}\right)\right) \\
& \geqslant-\sup \left(\mathbf{R} \operatorname{Hom}_{R}(P, Y)_{\mathfrak{p}}\right) \\
& \geqslant-\sup \left(\mathbf{R} \operatorname{Hom}_{R}(P, Y)\right)
\end{aligned}
$$

with the next sequence

$$
\inf \left(P_{\mathfrak{p}}\right) \leqslant \sup \left(P_{\mathfrak{p}}\right) \leqslant \sup (P)
$$

where the assumption $\operatorname{supp}_{R}(P)=\mathrm{V}(\mathfrak{a})$ is used.

(b) - (IC) Our assumptions on $P$ imply that $\inf (P), \sup (P) \in \mathbb{Z}$. Thus, the desired conclusions follow directly from part (国) because of Fact 2.8(国) -4]

See Theorem 3.11 for an improvement on our next result. It should be noted that the condition $\operatorname{supp}_{R}(Y) \subseteq \mathrm{V}(\mathfrak{a})$ is a bit strange to us: given the co-support formula in Fact 2.2. it would seem more natural to assume co-supp ${ }_{R}(Y) \subseteq \mathrm{V}(\mathfrak{a})$; see, however, Theorem 3.6 .

Theorem 3.4. Let $P \in \mathcal{D}_{b}(R)$ be a-adically finite such that $\operatorname{pd}_{R}(P)<\infty$ and $\operatorname{supp}_{R}(P)=\mathrm{V}(\mathfrak{a})$. Let $Y \in \mathcal{D}(R)$ be such that $\operatorname{supp}_{R}(Y) \subseteq \mathrm{V}(\mathfrak{a})$.

(a) One has $\sup (Y)-\sup (P)-n \leqslant \sup \left(\mathbf{R} \operatorname{Hom}_{R}(P, Y)\right)$.

(b) One has $Y \in \mathcal{D}_{-}(R)$ if and only if $\mathbf{R H o m}_{R}(P, Y) \in \mathcal{D}_{-}(R)$.

(c) One has $Y \simeq 0$ if and only if $\mathbf{R H o m}_{R}(P, Y) \simeq 0$.

Proof. (国) Set $K^{*}:=\mathbf{R} \operatorname{Hom}_{R}(K, R) \simeq \Sigma^{-n} K$, so we have

$$
\operatorname{pd}_{R}\left(K^{*}\right)=\operatorname{pd}_{R}(K)-n=0 .
$$

\footnotetext{
${ }^{4}$ The following alternate proof of part (IC) is worth noting. By Hom-evaluation 2, Lemma 4.4(I)], the assumptions on $P$ provide an isomorphism $\mathbf{R H o m}_{R}(P, Y) \simeq P^{*} \otimes_{R}^{\mathbf{L}} Y$ where $P^{*}=\mathbf{R H o m}_{R}(P, R)$ satisfies $\operatorname{supp}_{R}\left(P^{*}\right)=\operatorname{supp}_{R}(P)$, as in the proof of Corollary 4.3 below. Now apply Fact 2.2
} 
This explains the first step in the next display.

$$
\begin{aligned}
\sup \left(\mathbf{R H o m}_{R}(P, Y)\right) & =\operatorname{pd}_{R}\left(K^{*}\right)+\sup \left(\mathbf{R} \operatorname{Hom}_{R}(P, Y)\right) \\
& \geqslant \sup \left(K^{*} \otimes_{R}^{\mathbf{L}} \mathbf{R} \operatorname{Hom}_{R}(P, Y)\right) \\
& =\sup \left(\mathbf{R} \operatorname{Hom}_{R}\left(K \otimes_{R}^{\mathbf{L}} P, Y\right)\right) \\
& \geqslant \sup (Y)-\sup \left(K \otimes_{R}^{\mathbf{L}} P\right) \\
& \geqslant \sup (Y)-n-\sup (P)
\end{aligned}
$$

The second and last steps are by Fact 2.8(cic). The fourth step is from Lemma3.3(a), applied to the complex $K \otimes_{R}^{\mathbf{L}} P \in \mathcal{D}_{\mathrm{b}}^{\mathrm{f}}(R)$ which has $\operatorname{supp}_{R}\left(K \otimes_{R}^{\mathrm{L}} P\right)=\mathrm{V}(\mathfrak{a})$. The third step is from the evaluation and adjunction isomorphisms

$$
\begin{aligned}
K^{*} \otimes_{R}^{\mathbf{L}} \mathbf{R H o m}_{R}(P, Y) & \simeq \mathbf{R} \operatorname{Hom}_{R}\left(P, K^{*} \otimes_{R}^{\mathbf{L}} Y\right) \\
& \simeq \mathbf{R} \operatorname{Hom}_{R}\left(P, \mathbf{R} \operatorname{Hom}_{R}(K, Y)\right) \\
& \simeq \mathbf{R} \operatorname{Hom}_{R}\left(K \otimes_{R}^{\mathbf{L}} P, Y\right)
\end{aligned}
$$

see Fact 2.9

(b) - (IC) Note that our assumptions on $P$ imply that $0 \not f P \in \mathcal{D}_{\mathrm{b}}(R)$, so we have $\inf (P), \sup (P) \in \mathbb{Z}$. Thus, the desired conclusions follow directly from part (a) because of Fact 2.8(a).

Remark 3.5. As in 29, Theorem 3.4 has the following consequences. Let $P \in$ $\mathcal{D}_{\mathrm{b}}(R)$ be $\mathfrak{a}$-adically finite with $\operatorname{pd}_{R}(P)<\infty$ and $\mathrm{V}(\mathfrak{a})=\operatorname{supp}_{R}(P)$. Let $f: X \rightarrow Y$ be a morphism in $\mathcal{D}(R)$ with $\operatorname{supp}_{R}(X), \operatorname{supp}_{R}(Y) \subseteq \mathrm{V}(\mathfrak{a})$. Then $f$ is an isomorphism if and only if $\mathbf{R H o m}_{R}(P, f)$ is an isomorphism. We resist the urge to document every possible variation on this theme, here and elsewhere in the paper.

Each result like Theorem 3.4 has a version for $P \in \mathcal{D}_{\mathrm{b}}^{\mathrm{f}}(R)$ Like Lemma 3.3 While most of these results are new, we resist the urge to document them all, for the sake of brevity.

See Theorem 3.13 for an improvement of the next result.

Theorem 3.6. Let $P \in \mathcal{D}_{b}(R)$ be a-adically finite such that $\operatorname{pd}_{R}(P)<\infty$ and $\operatorname{supp}_{R}(P)=\mathrm{V}(\mathfrak{a})$. Let $Y \in \mathcal{D}(R)$ be such that $\operatorname{co-supp}(Y) \subseteq \mathrm{V}(\mathfrak{a})$.

(a) One has $\sup (Y)-\sup (P)-3 n \leqslant \sup \left(\mathbf{R} \operatorname{Hom}_{R}(P, Y)\right)$.

(b) One has $Y \in \mathcal{D}_{-}(R)$ if and only if $\mathbf{R H o m}_{R}(P, Y) \in \mathcal{D}_{-}(R)$.

Proof. It suffices to prove part (国). To this end, the first step in the next sequence is from Fact 2.8(c).

$$
\begin{aligned}
\sup \left(\mathbf{R H o m}_{R}(P, Y)\right) & \geqslant \sup \left(K \otimes_{R}^{\mathbf{L}} \mathbf{R} \operatorname{Hom}_{R}(P, Y)\right)-n \\
& =\sup \left(\mathbf{R} \operatorname{Hom}_{R}\left(P, K \otimes_{R}^{\mathbf{L}} Y\right)\right)-n \\
& \geqslant \sup \left(K \otimes_{R}^{\mathbf{L}} Y\right)-\sup (P)-2 n \\
& \geqslant \sup (Y)-\sup (P)-3 n
\end{aligned}
$$

The second step is by tensor-evaluation 2.9, and the third step follows from Theorem 3.4(国) since we have $\operatorname{supp}_{R}\left(K \otimes_{R}^{\mathrm{L}} Y\right) \subseteq \operatorname{supp}_{R}(K)=\mathrm{V}(\mathfrak{a})$. The last step is from Fact 2.11(国). 
Bounding by Tor. Here is a version of Propositions 3.1 3.2 for Tor. As with those results, the point is to guarantee the existence of non-zero Tor-modules, when one assumes enough boundedness on $X$ and $Y$ to guarantee that $X \otimes_{R}^{\mathrm{L}} Y \in \mathcal{D}_{+}(R)$.

Proposition 3.7. Let $X \in \mathcal{D}_{b}(R)$ be a-adically finite with $\operatorname{supp}_{R}(X)=\mathrm{V}(\mathfrak{a})$. Let $Y \in \mathcal{D}_{+}(R)$ be such that either $\operatorname{supp}_{R}(Y) \subseteq \mathrm{V}(\mathfrak{a})$ or $\operatorname{co-supp}_{R}(Y) \subseteq \mathrm{V}(\mathfrak{a})$.

(a) Then there is an inequality

$$
\inf \left(X \otimes_{R}^{\mathbf{L}} Y\right) \leqslant \sup (X)+\inf (Y)+2 n .
$$

(b) One has $Y \simeq 0$ if and only if $X \otimes_{R}^{\mathrm{L}} Y \simeq 0$.

Proof. (国) Let $E$ be a faithfully injective $R$-module and set $(-)^{\vee}:=\mathbf{R H o m}_{R}(-, E)$.

Assume first that $\operatorname{supp}_{R}(Y) \subseteq \mathrm{V}(\mathfrak{a})$. The first step in the following display is from Fact 2.8(c) since $\inf (K)=0$.

$$
\begin{aligned}
\inf \left(X \otimes_{R}^{\mathbf{L}} Y\right) & \leqslant \inf \left(X \otimes_{R}^{\mathbf{L}} Y \otimes_{R}^{\mathbf{L}} K\right) \\
& =-\sup \left(\left(X \otimes_{R}^{\mathbf{L}} Y \otimes_{R}^{\mathbf{L}} K\right)^{\vee}\right) \\
& =-\sup \left(\mathbf{R} \operatorname{Hom}_{R}\left(X,\left(Y \otimes_{R}^{\mathbf{L}} K\right)^{\vee}\right)\right) \\
& \leqslant-\sup \left(\left(Y \otimes_{R}^{\mathbf{L}} K\right)^{\vee}\right)+\sup (X)+n \\
& =\inf \left(Y \otimes_{R}^{\mathbf{L}} K\right)+\sup (X)+n \\
& \leqslant \inf (Y)+\sup (X)+2 n
\end{aligned}
$$

The second and fifth steps are from the faithful injectivity of $E$, and the third step is from adjointness. For the fourth step, note that $\mathfrak{a}$ annihilates the homology of $Y \otimes_{R}^{\mathbf{L}} K$, so it also annihilates the homology of $\left(Y \otimes_{R}^{\mathbf{L}} K\right)^{\vee}$; it follows that $\operatorname{supp}_{R}\left(\left(Y \otimes_{R}^{\mathbf{L}} K\right)^{\vee}\right) \subseteq \mathrm{V}(\mathfrak{a})$. Since we also have $\left(Y \otimes_{R}^{\mathbf{L}} K\right)^{\vee} \in \mathcal{D}_{-}(R)$, the fourth step follows from Proposition 3.1(国). The sixth step is from Fact 2.10(a).

Assume next that $\operatorname{co}^{-\operatorname{supp}_{R}}(Y) \subseteq \mathrm{V}(\mathfrak{a})$. The first step in the next display is from Fact 2.8(C).

$$
\begin{aligned}
\inf \left(X \otimes_{R}^{\mathbf{L}} Y\right) & \leqslant \inf \left(X \otimes_{R}^{\mathbf{L}} Y \otimes_{R}^{\mathbf{L}} K\right) \\
& \leqslant \inf \left(Y \otimes_{R}^{\mathbf{L}} K\right)+\sup (X)+2 n \\
& =\inf (Y)+\sup (X)+2 n
\end{aligned}
$$

Since $\operatorname{supp}_{R}\left(K \otimes_{R}^{\mathbf{L}} Y\right) \subseteq \operatorname{supp}_{R}(K) \subseteq \mathrm{V}(\mathfrak{a})$, the second step follows from the previous paragraph. The third step is from Fact 2.11(国).

(b) This follows from part (回), using Fact 2.8(b).

We continue with more results along the lines of Theorem 1.1 See Theorem 3.16 below for an improvement.

Theorem 3.8. Let $F \in \mathcal{D}_{b}(R)$ be a-adically finite such that $\operatorname{fd}_{R}(F)<\infty$ and $\mathrm{V}(\mathfrak{a})=\operatorname{supp}_{R}(F)$. Let $Z \in \mathcal{D}(R)$ be such that co-supp $R(Z) \subseteq \mathrm{V}(\mathfrak{a})$.

(a) One has $\inf \left(F \otimes_{R}^{\mathbf{L}} Z\right) \leqslant \inf (Z)+\sup (F)+n$.

(b) One has $Z \in \mathcal{D}_{+}(R)$ if and only if $F \otimes_{R}^{\mathrm{L}} Z \in \mathcal{D}_{+}(R)$.

(c) One has $Z \simeq 0$ if and only if $F \otimes_{R}^{\mathrm{L}} Z \simeq 0$.

Proof. (国) We first prove the special case where $\mathfrak{a} \mathrm{H}(Z)=0$. Let $E$ be a faithfully injective $R$-module, and set $(-)^{\vee}:=\mathbf{R H o m}_{R}(-, E)$. The assumption $\mathfrak{a} \mathrm{H}(Z)=0$ implies that $\mathfrak{a} H\left(Z^{\vee}\right)=0$ since $E$ is injective. In particular, we have $\operatorname{supp}_{R}\left(Z^{\vee}\right) \subseteq$ 
$\mathrm{V}(\mathfrak{a})$ by Fact 2.2 . The fact that $E$ is faithfully injective explains the first and last steps in the next sequence.

$$
\begin{aligned}
\inf \left(F \otimes_{R}^{\mathbf{L}} Z\right) & =-\sup \left(\left(F \otimes_{R}^{\mathbf{L}} Z\right)^{\vee}\right) \\
& =-\sup \left(\mathbf{R} \operatorname{Hom}_{R}\left(F, Z^{\vee}\right)\right) \\
& \leqslant n+\sup (F)-\sup \left(Z^{\vee}\right) \\
& =n+\sup (F)+\inf (Z)
\end{aligned}
$$

The second step is from Hom-tensor adjointness. The third step is from Theorem 3.4(国), since [27, Theorem 6.1] implies $\operatorname{pd}_{R}(F)<\infty$.

Now we deal with the general case. The complex $Z \otimes_{R}^{\mathbf{L}} K$ satisfies $\mathfrak{a} \mathrm{H}\left(Z \otimes_{R}^{\mathbf{L}} K\right)=$ 0 . Thus, the previous paragraph explains the second step in the next sequence.

$$
\begin{aligned}
\inf \left(F \otimes_{R}^{\mathbf{L}} Z\right) & \leqslant \inf \left(F \otimes_{R}^{\mathbf{L}} Z \otimes_{R}^{\mathbf{L}} K\right) \\
& \leqslant n+\sup (F)+\inf \left(Z \otimes_{R}^{\mathbf{L}} K\right) \\
& =n+\sup (F)+\inf (Z)
\end{aligned}
$$

The first step is from Fact 2.8( (c), and the third step is from Fact 2.11(国), wherein our co-support assumption is used.

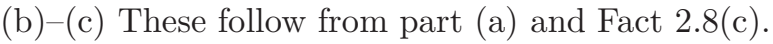

See Theorem 3.15 for an improvement of the next result.

Theorem 3.9. Let $F \in \mathcal{D}_{b}(R)$ be a-adically finite such that $\operatorname{fd}_{R}(F)<\infty$ and $\mathrm{V}(\mathfrak{a})=\operatorname{supp}_{R}(F)$. Let $Z \in \mathcal{D}(R)$ be such that $\operatorname{supp}_{R}(Z) \subseteq \mathrm{V}(\mathfrak{a})$.

(a) One has $\inf \left(F \otimes_{R}^{\mathbf{L}} Z\right) \leqslant \inf (Z)+\sup (F)+2 n$.

(b) One has $Z \in \mathcal{D}_{+}(R)$ if and only if $F \otimes_{R}^{\mathrm{L}} Z \in \mathcal{D}_{+}(R)$.

Proof. It suffices to prove part (a)

The complex $Z \otimes_{R}^{\mathbf{L}} K$ satisfies $\mathfrak{a} \mathrm{H}\left(Z \otimes_{R}^{\mathbf{L}} K\right)=0$. Thus, Theorem 3.8(a) explains the second step in the next sequence.

$$
\begin{aligned}
\inf \left(F \otimes_{R}^{\mathbf{L}} Z\right) & \leqslant \inf \left(F \otimes_{R}^{\mathbf{L}} Z \otimes_{R}^{\mathbf{L}} K\right) \\
& \leqslant n+\sup (F)+\inf \left(Z \otimes_{R}^{\mathbf{L}} K\right) \\
& \leqslant 2 n+\sup (F)+\inf (Z)
\end{aligned}
$$

The first and third steps are from Facts 2.8([) and 2.10(国).

Ext Revisited. Each of the preceding results of this section deals with only one invariant, either $\sup \left(\mathbf{R} \operatorname{Hom}_{R}(P, Y)\right)$ or $\inf \left(F \otimes_{R}^{\mathbf{L}} Z\right)$. We now show how to use these results to bootstrap our way to other invariants, e.g., $\inf \left(\mathbf{R H o m}_{R}(P, Y)\right)$ and $\sup \left(F \otimes_{R}^{\mathbf{L}} Z\right)$, beginning with an improvement of Theorem 3.4.

Lemma 3.10. Let $P \in \mathcal{D}_{b}(R)$ be $\mathfrak{a}$-adically finite such that $p \not 00$ and $\operatorname{pd}_{R}(P)<\infty$. Let $Y \in \mathcal{D}(R)$ be such that $\operatorname{supp}_{R}(Y) \subseteq \operatorname{supp}_{R}(P)$.

(a) There are inequalities

$$
\begin{gathered}
\inf \left(\mathbf{R} \operatorname{Hom}_{R}(P, Y)\right) \leqslant \inf (Y)-\inf (P)+2 n \\
\sup (Y)-\sup (P) \leqslant \sup \left(\mathbf{R} \operatorname{Hom}_{R}(P, Y)\right) \\
\operatorname{amp}(Y)-\operatorname{amp}(P)-2 n \leqslant \operatorname{amp}\left(\mathbf{R H o m}_{R}(P, Y)\right) .
\end{gathered}
$$

(b) For $* \in\{+,-, b\}$, one has $Y \in \mathcal{D}_{*}(R)$ if and only if $\mathbf{R H o m}_{R}(P, Y) \in \mathcal{D}_{*}(R)$. 
Proof. In light of Fact 2.8 and Lemma3.3(国), it suffices to prove the first inequality in part (国). Set $P^{*}:=\mathbf{R H o m}_{R}(P, R)$ which is in $\mathcal{D}_{\mathrm{b}}^{\mathrm{f}}(R)$ and has $\operatorname{pd}_{R}\left(P^{*}\right)<\infty$. Also, from Fact 2.8(c), we have $\sup \left(P^{*}\right) \leqslant-\inf (P)$, and thus the last step in the next sequence.

$$
\begin{aligned}
\inf (Y) & \geqslant \inf \left(Y \otimes_{R}^{\mathbf{L}} K\right)-n \\
& \geqslant \inf \left(P^{*} \otimes_{R}^{\mathbf{L}} Y \otimes_{R}^{\mathbf{L}} K\right)-\sup \left(P^{*}\right)-2 n \\
& \geqslant \inf \left(P^{*} \otimes_{R}^{\mathbf{L}} Y\right)-\sup \left(P^{*}\right)-2 n \\
& =\inf \left(\mathbf{R H o m}{ }_{R}(P, Y)\right)-\sup \left(P^{*}\right)-2 n \\
& \geqslant \inf \left(\mathbf{R H o m}_{R}(P, Y)\right)+\inf (P)-2 n
\end{aligned}
$$

The first step is from Fact 2.10(可). The second step is by Theorem 3.8(a), since $\mathfrak{a}$ annihilates $\mathrm{H}\left(Y \otimes_{R}^{\mathbf{L}} K\right)$. The third step is from Fact 2.8(ㄷ), and the fourth step is from the isomorphisms $P^{*} \otimes_{R}^{\mathrm{L}} Y \simeq \mathbf{R H o m}_{R}\left(P^{* *}, Y\right) \simeq \mathbf{R H o m}_{R}(P, Y)$; see [2, Lemma 4.4(I)].

Theorem 3.11. Let $P \in \mathcal{D}_{b}(R)$ be a-adically finite such that $\operatorname{pd}_{R}(P)<\infty$ and $\operatorname{supp}_{R}(P)=\mathrm{V}(\mathfrak{a})$. Let $Y \in \mathcal{D}(R)$ be such that $\operatorname{supp}_{R}(Y) \subseteq \mathrm{V}(\mathfrak{a})$.

(a) There are inequalities

$$
\begin{gathered}
\inf \left(\mathbf{R H o m}_{R}(P, Y)\right) \leqslant \inf (Y)-\inf (P)+3 n \\
\sup (Y)-\sup (P)-n \leqslant \sup \left(\mathbf{R} \operatorname{Hom}_{R}(P, Y)\right) \\
\operatorname{amp}(Y)-\operatorname{amp}(P)-4 n \leqslant \operatorname{amp}\left(\mathbf{R H o m}_{R}(P, Y)\right) .
\end{gathered}
$$

(b) For $* \in\{+,-, b\}$, one has $Y \in \mathcal{D}_{*}(R)$ if and only if $\mathbf{R H o m}_{R}(P, Y) \in \mathcal{D}_{*}(R)$.

Proof. In light of Fact 2.8 and Theorem 3.4(国), it suffices to prove the first inequality in part (国). To this end, we apply Lemma 3.10(国) to the complex $\mathbf{R H o m}_{R}(K, P) \in$ $\mathcal{D}_{\mathrm{b}}^{\mathrm{f}}(R)$. For this, note that $\operatorname{pd}_{R}\left(\mathbf{R H o m}_{R}(K, P)\right)<\infty$ and $\mathbf{R H o m}_{R}(K, P) \simeq$ $\Sigma^{-n} K \otimes_{R}^{\mathrm{L}} P$ by Fact 2.9. It follows by assumption that

$$
\operatorname{supp}_{R}\left(\mathbf{R H o m}_{R}(K, P)\right)=\operatorname{supp}_{R}\left(K \otimes_{R}^{\mathbf{L}} P\right)=\operatorname{supp}_{R}(K) \bigcap \operatorname{supp}_{R}(P)=\mathrm{V}(\mathfrak{a}) .
$$

Thus, Lemma 3.10(国) explains the first step in the next display.

$$
\begin{aligned}
\inf (Y) & \geqslant \inf \left(\mathbf{R} \operatorname{Hom}_{R}\left(\mathbf{R H o m}_{R}(K, P), Y\right)\right)+\inf \left(\mathbf{R} \operatorname{Hom}_{R}(K, P)\right)-2 n \\
& =\inf \left(K \otimes_{R}^{\mathbf{L}} \mathbf{R} \operatorname{Hom}_{R}(P, Y)\right)+\inf \left(\Sigma^{-n} K \otimes_{R}^{\mathbf{L}} P\right)-2 n \\
& =\inf \left(K \otimes_{R}^{\mathbf{L}} \mathbf{R} \operatorname{Hom}_{R}(P, Y)\right)+\inf \left(K \otimes_{R}^{\mathbf{L}} P\right)-3 n \\
& \geqslant \inf \left(\mathbf{R} \operatorname{Hom}_{R}(P, Y)\right)+\inf (P)-3 n
\end{aligned}
$$

The second step is from Hom-evaluation 2.9 and the isomorphism $\mathbf{R H o m}_{R}(K, P) \simeq$ $\Sigma^{-n} K \otimes_{R}^{\mathbf{L}} P$ noted above. The third step is straightforward, and the fourth one is from Fact 2.8(c).

The next result follows directly from Theorems 3.4 and 3.11 in the special case $F=\mathbf{R} \Gamma_{\mathfrak{a}}(R)$. In it we use the notation $\operatorname{cd}_{\mathfrak{a}}(R):=-\inf \left(\mathbf{R} \Gamma_{\mathfrak{a}}(R)\right)$, which is the cohomological dimension of $R$ with respect to $\mathfrak{a}$ and the standard interpretation $\operatorname{depth}_{\mathfrak{a}}(R)=-\sup \left(\mathbf{R} \Gamma_{\mathfrak{a}}(R)\right)$ of depth in terms of local cohomology.

Corollary 3.12. Let $Y \in \mathcal{D}(R)$ be such that $\operatorname{supp}_{R}(Y) \subseteq \mathrm{V}(\mathfrak{a})$. 
(a) There are inequalities

$$
\begin{gathered}
\inf \left(\mathbf{L} \Lambda^{\mathfrak{a}}(Y)\right) \leqslant \inf (Y)+\operatorname{cd}_{\mathfrak{a}}(R)+3 n \\
\sup (Y)+\operatorname{depth}_{\mathfrak{a}}(R)-n \leqslant \sup \left(\mathbf{L} \Lambda^{\mathfrak{a}}(Y)\right) \\
\operatorname{amp}(Y)+\operatorname{depth}_{\mathfrak{a}}(R)-\operatorname{cd}_{\mathfrak{a}}(R)-4 n \leqslant \operatorname{amp}\left(\mathbf{L} \Lambda^{\mathfrak{a}}(Y)\right) .
\end{gathered}
$$

(b) For $* \in\{+,-, b\}$, one has $Y \in \mathcal{D}_{*}(R)$ if and only if $\mathbf{L} \Lambda^{\mathfrak{a}}(Y) \in \mathcal{D}_{*}(R)$.

(c) One has $Y \simeq 0$ if and only if $\mathbf{L} \Lambda^{\mathfrak{a}}(Y) \simeq 0$.

Next, we improve on Theorem 3.6

Theorem 3.13. Let $P \in \mathcal{D}_{b}(R)$ be a-adically finite such that $\operatorname{pd}_{R}(P)<\infty$ and $\operatorname{supp}_{R}(P)=\mathrm{V}(\mathfrak{a})$. Let $Y \in \mathcal{D}(R)$ be such that co-supp $(Y) \subseteq \mathrm{V}(\mathfrak{a})$.

(a) There are inequalities

$$
\begin{gathered}
\inf \left(\mathbf{R H o m}_{R}(P, Y)\right) \leqslant \inf (Y)-\inf (P)+3 n \\
\sup (Y)-\sup (P)-3 n \leqslant \sup \left(\mathbf{R} \operatorname{Hom}_{R}(P, Y)\right) \\
\operatorname{amp}(Y)-\operatorname{amp}(P)-6 n \leqslant \operatorname{amp}\left(\mathbf{R H o m}_{R}(P, Y)\right) .
\end{gathered}
$$

(b) For $* \in\{+,-, b\}$, one has $Y \in \mathcal{D}_{*}(R)$ if and only if $\mathbf{R H o m}_{R}(P, Y) \in \mathcal{D}_{*}(R)$.

Proof. Because of Theorem3.6(包), we need only prove the first inequality of part (国). Since we have co-supp ${ }_{R}(\mathbf{R H o m} R(P, Y)) \subseteq \operatorname{supp}_{R}(P)=\mathrm{V}(\mathfrak{a})$, the first step in the next sequence is from Fact 2.11(国).

$$
\begin{aligned}
\inf \left(\mathbf{R H o m}_{R}(P, Y)\right) & =\inf \left(K \otimes_{R}^{\mathbf{L}} \mathbf{R} \operatorname{Hom}_{R}(P, Y)\right) \\
& =\inf \left(\mathbf{R} \operatorname{Hom}_{R}\left(P, K \otimes_{R}^{\mathbf{L}} Y\right)\right) \\
& \leqslant \inf \left(K \otimes_{R}^{\mathbf{L}} Y\right)-\inf (P)+3 n \\
& =\inf (Y)-\inf (P)+3 n
\end{aligned}
$$

The second step is by tensor-evaluation 2.9 and the third step follows from Theorem 3.11(国) since we have $\operatorname{supp}_{R}\left(K \otimes_{R}^{\mathbf{L}} Y\right) \subseteq \operatorname{supp}_{R}(K)=\mathrm{V}(\mathfrak{a})$. The last step is from Fact 2.11(国).

Remark 3.14. Note that we do not bother to state the special case $F=\mathbf{R} \Gamma_{\mathfrak{a}}(R)$ of Theorem 3.13, in contrast with Corollary 3.12 The reason is that Theorem 3.13 is trivial in this case. Indeed, the assumption co-supp ${ }_{R}(Y) \subseteq \mathrm{V}(\mathfrak{a})$ implies that $Y \simeq$ $\mathbf{L} \Lambda^{\mathfrak{a}}(Y) \simeq \mathbf{R H o m}_{R}\left(\mathbf{R} \Gamma_{\mathfrak{a}}(R), Y\right)$ by Fact 2.2. Thus, for instance Theorem 3.13(b) says that for $* \in\{+,-, \mathrm{b}\}$, one has $Y \in \mathcal{D}_{*}(R)$ if and only if $Y \in \mathcal{D}_{*}(R)$.

Tor Revisited. Our next result improves upon Theorem 3.9 and contains Theorem 1.2 from the introduction.

Theorem 3.15. Let $F \in \mathcal{D}_{b}(R)$ be a-adically finite such that $\operatorname{fd}_{R}(F)<\infty$ and $\operatorname{supp}_{R}(F)=\mathrm{V}(\mathfrak{a})$. Let $Z \in \mathcal{D}(R)$ be such that $\operatorname{supp}_{R}(Z) \subseteq \mathrm{V}(\mathfrak{a})$.

(a) There are inequalities

$$
\begin{gathered}
\inf \left(F \otimes_{R}^{\mathbf{L}} Z\right) \leqslant \inf (Z)+\sup (F)+2 n \\
\inf (F)+\sup (Z)-3 n \leqslant \sup \left(F \otimes_{R}^{\mathbf{L}} Z\right) \\
\operatorname{amp}(Z)-\operatorname{amp}(F)-5 n \leqslant \operatorname{amp}\left(F \otimes_{R}^{\mathbf{L}} Z\right) .
\end{gathered}
$$

(b) For $* \in\{+,-, b\}$, one has $Z \in \mathcal{D}_{*}(R)$ if and only if $F \otimes \mathbb{R}_{R}^{\mathbf{L}} Z \in \mathcal{D}_{*}(R)$. 
Proof. Recall that [27, Theorem 6.1] implies $\operatorname{pd}_{R}(F)<\infty$. Let $E$ be a faithfully injective $R$-module, and set $(-)^{\vee}:=\mathbf{R} \operatorname{Hom}_{R}(-, E)$. It follows from Fact 2.2 that $\mathrm{co-Supp}_{R}\left(Z^{\vee}\right) \subseteq \operatorname{supp}_{R}(Z) \subseteq \mathrm{V}(\mathfrak{a})$. Thus, the third step in the next display is from Theorem 3.13 (a) .

$$
\begin{aligned}
\sup \left(F \otimes_{R}^{\mathbf{L}} Z\right) & =-\inf \left(\left(F \otimes_{R}^{\mathbf{L}} Z\right)^{\vee}\right) \\
& =-\inf \left(\mathbf{R} \operatorname{Hom}_{R}\left(F, Z^{\vee}\right)\right) \\
& \geqslant \inf (F)-\inf \left(Z^{\vee}\right)-3 n \\
& \geqslant \inf (F)+\sup (Z)-3 n
\end{aligned}
$$

The first and last steps follow from the fact that $E$ is faithfully injective, and the second step is from Hom-tensor adjointness. This explains the second inequality of part (国). The rest of the result follows from Theorem 3.9.

To conclude this section, we improve on Theorem 3.8, the corollary is the special case $F=\mathbf{R} \Gamma_{\mathfrak{a}}(R)$.

Theorem 3.16. Let $F \in \mathcal{D}_{b}(R)$ be a-adically finite such that $\operatorname{fd}_{R}(F)<\infty$ and $\operatorname{supp}_{R}(F)=\mathrm{V}(\mathfrak{a})$. Let $Z \in \mathcal{D}(R)$ be such that $\operatorname{co-supp}(Z) \subseteq \mathrm{V}(\mathfrak{a})$.

(a) There are inequalities

$$
\begin{gathered}
\inf \left(F \otimes_{R}^{\mathbf{L}} Z\right) \leqslant \inf (Z)+\sup (F)+n \\
\sup (Z)+\inf (F)-5 n \leqslant \sup \left(F \otimes_{R}^{\mathbf{L}} Z\right) \\
\operatorname{amp}(Z)-\operatorname{amp}(F)-6 n \leqslant \operatorname{amp}\left(F \otimes_{R}^{\mathbf{L}} Z\right) .
\end{gathered}
$$

(b) For $* \in\{+,-, b\}$, one has $Z \in \mathcal{D}_{*}(R)$ if and only if $F \otimes_{R}^{\mathrm{L}} Z \in \mathcal{D}_{*}(R)$.

Proof. In light of Theorem 3.8 we need only bound $\sup \left(F \otimes_{R}^{\mathbf{L}} Z\right)$. We compute, starting with an application of Fact 2.10(或).

$$
\begin{aligned}
\sup \left(F \otimes_{R}^{\mathrm{L}} Z\right) & =\sup \left(F \otimes_{R}^{\mathrm{L}} Z \otimes_{R}^{\mathrm{L}} K\right)-n \\
& \geqslant \inf (F)+\sup \left(Z \otimes_{R}^{\mathrm{L}} K\right)-4 n \\
& \geqslant \inf (F)+\sup (Z)-5 n
\end{aligned}
$$

The remaining steps are by Theorem 3.15(国) and Fact 2.11(国).

Corollary 3.17. Let $Z \in \mathcal{D}(R)$ be such that $\operatorname{co}^{-\operatorname{supp}_{R}}(Z) \subseteq \mathrm{V}(\mathfrak{a})$.

(a) There are inequalities

$$
\begin{gathered}
\inf \left(\mathbf{R} \Gamma_{\mathfrak{a}}(Z)\right) \leqslant \inf (Z)-\operatorname{depth}_{\mathfrak{a}}(R)+n \\
\sup (Z)-\operatorname{cd}_{\mathfrak{a}}(R)-5 n \leqslant \sup \left(\mathbf{R} \Gamma_{\mathfrak{a}}(Z)\right) \\
\operatorname{amp}(Z)-\operatorname{cd}_{\mathfrak{a}}(R)+\operatorname{depth}_{\mathfrak{a}}(R)-6 n \leqslant \operatorname{amp}\left(\mathbf{R} \Gamma_{\mathfrak{a}}(Z)\right) .
\end{gathered}
$$

(b) For $* \in\{+,-, b\}$, one has $Z \in \mathcal{D}_{*}(R)$ if and only if $\mathbf{R} \Gamma_{\mathfrak{a}}(Z) \in \mathcal{D}_{*}(R)$.

(c) One has $Z \simeq 0$ if and only if $\mathbf{R} \Gamma_{\mathfrak{a}}(Z) \simeq 0$.

\section{Bounding Homology II: Over a Ring Homomorphism}

We continue with the theme from the previous section. For instance, the next result, containing Theorem 1.1 from the introduction, is similar to Theorem 3.15, the differences being fewer restrictions on $F$ and more restrictions on $X$. For perspective on the assumption $\varphi^{*}\left(\operatorname{supp}_{S}(F)\right) \supseteq \mathrm{V}(\mathfrak{a}) \bigcap \mathrm{m}-\operatorname{Spec}(R)$, note that 27, Proposition 5.6] implies that this condition is less restrictive than the seemingly more 
natural condition $\operatorname{supp}_{R}(F) \supseteq \mathrm{V}(\mathfrak{a}) \bigcap \mathrm{m}-\operatorname{Spec}(R)$. Also, technically, the complex $X \otimes_{R}^{\mathbf{L}} F$ in the next result should be written $X \otimes_{R}^{\mathbf{L}} Q(F)$ where $Q: \mathcal{D}(S) \rightarrow \mathcal{D}(R)$ is the forgetful functor, and similarly for $\operatorname{fd}_{R}(F)$. We choose not to do this, in order to avoid cumbersome notation.

Theorem 4.1. Let $\varphi: R \rightarrow S$ be a ring homomorphism such that $\mathfrak{a} S \neq S$, and let $F \in \mathcal{D}_{b}(S)$ be a $S$-adically finite such that $\operatorname{fd}_{R}(F)<\infty$ and $\varphi^{*}\left(\operatorname{supp}_{S}(F)\right) \supseteq$ $\mathrm{V}(\mathfrak{a}) \cap \mathrm{m}-\operatorname{Spec}(R)$. Let $X \in \mathcal{D}(R)$ be such that $\operatorname{supp}_{R}(X) \subseteq \mathrm{V}(\mathfrak{a})$. Assume that $K \otimes_{R}^{\mathbf{L}} X \in \mathcal{D}^{f}(R)$.

(a) There are inequalities

$$
\begin{gathered}
\inf \left(F \otimes_{R}^{\mathbf{L}} X\right) \leqslant \inf (X)+\sup (F)+2 n \\
\sup (X)+\inf (F)-n \leqslant \sup \left(F \otimes_{R}^{\mathbf{L}} X\right) \\
\operatorname{amp}(X)-\operatorname{amp}(F)-3 n \leqslant \operatorname{amp}\left(F \otimes_{R}^{\mathbf{L}} X\right) .
\end{gathered}
$$

(b) One has $X \simeq 0$ if and only if $F \otimes_{R}^{\mathbf{L}} X \simeq 0$.

(c) For each $* \in\{+,-, b\}$, one has $X \in \mathcal{D}_{*}(R)$ if and only if $F \otimes_{R}^{\mathbf{L}} X \in \mathcal{D}_{*}(R)$.

Proof. (a) We first treat the case where $F \in \mathcal{D}_{\mathrm{b}}^{\mathrm{f}}(S)$ and $X \in \mathcal{D}^{\mathrm{f}}(R)$. Since $F$ is in $\mathcal{D}_{\mathrm{b}}^{\mathrm{f}}(S)$, we have $\operatorname{supp}_{S}(F)=\operatorname{Supp}_{S}(F)$. Also, by [29, Proposition 3.15(a)] we know that $\operatorname{Supp}_{R}(X) \subseteq \mathrm{V}(\mathfrak{a})$. Thus, from the proof of [11, Theorem 4.2], we have

$$
\begin{gathered}
\inf \left(F \otimes_{R}^{\mathbf{L}} X\right) \leqslant \inf (X)+\sup (F) \\
\sup (X)+\inf (F) \leqslant \sup \left(F \otimes_{R}^{\mathbf{L}} X\right) \\
\operatorname{amp}(X)-\operatorname{amp}(F) \leqslant \operatorname{amp}\left(F \otimes_{R}^{\mathbf{L}} X\right) .
\end{gathered}
$$

For the general case, set $K^{S}:=S \otimes_{R}^{\mathrm{L}} K$. We apply the previous case to the complexes $K^{S} \otimes_{S}^{\mathbf{L}} F$ and $K \otimes_{R}^{\mathbf{L}} X$. For this, we need to check the support conditions for these complexes. For the first one, we have

$$
\operatorname{supp}_{S}\left(K^{S} \otimes_{S}^{\mathrm{L}} F\right)=\mathrm{V}(\mathfrak{a} S) \bigcap \operatorname{supp}_{S}(F)
$$

by Fact 2.2. so the condition $\varphi^{*}\left(\operatorname{supp}_{S}(F)\right) \supseteq \mathrm{V}(\mathfrak{a}) \cap \mathrm{m}-\operatorname{Spec}(R)$ implies that

$$
\varphi^{*}\left(\operatorname{supp}_{S}\left(K^{S} \otimes_{S}^{\mathbf{L}} F\right)\right) \supseteq \mathrm{V}(\mathfrak{a}) \bigcap \mathrm{m}-\operatorname{Spec}(R) .
$$

The condition $\operatorname{supp}_{R}\left(K \otimes_{R}^{\mathrm{L}} X\right) \subseteq \mathrm{V}(\mathfrak{a})$ is even easier.

The first inequality in the next sequence is from Fact 2.8(c).

$$
\begin{aligned}
\inf \left(F \otimes_{R}^{\mathbf{L}} X\right) & \leqslant \inf \left(K^{S} \otimes_{S}^{\mathbf{L}}\left(F \otimes_{R}^{\mathbf{L}} X\right) \otimes_{R}^{\mathbf{L}} K\right) \\
& =\inf \left(\left(K^{S} \otimes_{S}^{\mathbf{L}} F\right) \otimes_{R}^{\mathbf{L}}\left(K \otimes_{R}^{\mathbf{L}} X\right)\right) \\
& \leqslant \sup \left(K^{S} \otimes_{S}^{\mathbf{L}} F\right)+\inf \left(K \otimes_{R}^{\mathbf{L}} X\right) \\
& \leqslant 2 n+\sup (F)+\inf (X)
\end{aligned}
$$

The second step is from the isomorphism

$$
K^{S} \otimes_{S}^{\mathbf{L}}\left(F \otimes_{R}^{\mathbf{L}} X\right) \otimes_{R}^{\mathbf{L}} K \simeq\left(K^{S} \otimes_{S}^{\mathbf{L}} F\right) \otimes_{R}^{\mathbf{L}}\left(K \otimes_{R}^{\mathbf{L}} X\right)
$$

and the third step is from the first paragraph of this proof. The fourth step is a combination of Facts 2.8(c) and 2.10(可). This explains the inequality (4.1.1), 
while (4.1.2) follows similarly:

$$
\begin{aligned}
\inf (F)+n+\sup (X) & \leqslant \inf \left(K^{S} \otimes_{S}^{\mathbf{L}} F\right)+\sup \left(K \otimes_{R}^{\mathbf{L}} X\right) \\
& \leqslant \sup \left(\left(K^{S} \otimes_{S}^{\mathbf{L}} F\right) \otimes_{R}^{\mathbf{L}}\left(K \otimes_{R}^{\mathbf{L}} X\right)\right) \\
& =\sup \left(K^{S} \otimes_{S}^{\mathbf{L}}\left(F \otimes_{R}^{\mathbf{L}} X\right) \otimes_{R}^{\mathbf{L}} K\right) \\
& \leqslant 2 n+\sup \left(F \otimes_{R}^{\mathbf{L}} X\right) .
\end{aligned}
$$

The remaining conclusions from the statement of the theorem follow readily.

Next, we have a version of Theorem 4.1 for co-support. It is proved like Theorem 4.1 using Fact 2.11(a) instead of 2.10(a).

Theorem 4.2. Let $\varphi: R \rightarrow S$ be a ring homomorphism such that $\mathfrak{a} S \neq S$, and let $F \in \mathcal{D}_{b}(S)$ be $\mathfrak{a} S$-adically finite such that $\operatorname{fd}_{R}(F)<\infty$ and $\varphi^{*}\left(\operatorname{supp}_{S}(F)\right) \supseteq$ $\mathrm{V}(\mathfrak{a}) \bigcap \mathrm{m}-\operatorname{Spec}(R)$. Let $X \in \mathcal{D}(R)$ be such that $\operatorname{co}^{-\operatorname{supp}_{R}}(X) \subseteq \mathrm{V}(\mathfrak{a})$. Assume that $K \otimes_{R}^{\mathbf{L}} X \in \mathcal{D}^{f}(R)$.

(a) There are inequalities

$$
\begin{gathered}
\inf \left(F \otimes_{R}^{\mathbf{L}} X\right) \leqslant \inf (X)+\sup (F)+n \\
\sup (X)+\inf (F)-3 n \leqslant \sup \left(F \otimes_{R}^{\mathbf{L}} X\right) \\
\operatorname{amp}(X)-\operatorname{amp}(F)-4 n \leqslant \operatorname{amp}\left(F \otimes_{R}^{\mathbf{L}} X\right) \leqslant \operatorname{amp}(X)+\operatorname{fd}_{R}(F)-\inf (F) .
\end{gathered}
$$

(b) One has $X \simeq 0$ if and only if $F \otimes_{R}^{\mathbf{L}} X \simeq 0$.

(c) For each $* \in\{+,-, b\}$, one has $X \in \mathcal{D}_{*}(R)$ if and only if $F \otimes_{R}^{\mathbf{L}} X \in \mathcal{D}_{*}(R)$.

Ext Re-revisited. The previous two theorems yield more results about RHom. We begin with two results for $P \in \mathcal{D}_{\mathrm{b}}^{\mathrm{f}}(R)$. These are extended to the adically finite setting in Theorems 4.5 and 4.6 below.

Corollary 4.3. Let $P \in \mathcal{D}_{b}^{f}(R)$ be such that $\operatorname{supp}_{R}(P) \supseteq \mathrm{V}(\mathfrak{a}) \bigcap \mathrm{m}-\operatorname{Spec}(R)$ and $\operatorname{pd}_{R}(P)<\infty$. Let $X \in \mathcal{D}(R)$ be such that $\operatorname{supp}_{R}(X) \subseteq \mathrm{V}(\mathfrak{a})$ and $K \otimes_{R}^{\mathrm{L}} X \in \mathcal{D}^{f}(R)$.

(a) There are inequalities

$$
\begin{gathered}
\inf \left(\mathbf{R} \operatorname{Hom}_{R}(P, X)\right) \leqslant \inf (X)-\inf (P)+2 n \\
\sup (X)-\operatorname{pd}_{R}(P)-n \leqslant \sup \left(\mathbf{R} \operatorname{Hom}_{R}(P, X)\right) \\
\operatorname{amp}(X)-\operatorname{pd}_{R}(P)+\inf (P)-3 n \leqslant \operatorname{amp}\left(\mathbf{R H o m}_{R}(P, X)\right) .
\end{gathered}
$$

(b) One has $X \simeq 0$ if and only if $\mathbf{R} \operatorname{Hom}_{R}(P, X) \simeq 0$.

(c) For $* \in\{+,-, b\}$, one has $X \in \mathcal{D}_{*}(R)$ if and only if $\mathbf{R H o m}_{R}(P, X) \in \mathcal{D}_{*}(R)$.

Proof. Set $P^{*}:=\mathbf{R H o m}_{R}(P, R)$. The assumptions $P \in \mathcal{D}_{\mathrm{b}}^{\mathrm{f}}(R)$ and $\operatorname{pd}_{R}(P)<\infty$ imply that $P \simeq \operatorname{RHom}_{R}\left(P^{*}, R\right)$. In particular, we have $P \simeq 0$ if and only if $P^{*} \simeq 0$. For any prime ideal $\mathfrak{p} \in \operatorname{Spec}(R)$, it follows that $P_{\mathfrak{p}} \simeq 0$ if and only if $\left(P^{*}\right)_{\mathfrak{p}} \simeq 0$, so we have $\operatorname{Supp}_{R}(P)=\operatorname{Supp}_{R}\left(P^{*}\right)$, that is, $\operatorname{supp}_{R}(P)=\operatorname{supp}_{R}\left(P^{*}\right)$ since $P, P^{*} \in \mathcal{D}_{\mathrm{b}}^{\mathrm{f}}(R)$.

In the next sequence of isomorphisms, the second step is Hom-evaluation 2, Lemma 4.4(I)]

$\mathbf{R H o m}_{R}(P, X) \simeq \mathbf{R H o m}_{R}\left(\mathbf{R} \operatorname{Hom}_{R}\left(P^{*}, R\right), X\right) \simeq P^{*} \otimes_{R}^{\mathbf{L}} \mathbf{R} \operatorname{Hom}_{R}(R, X) \simeq P^{*} \otimes_{R}^{\mathbf{L}} X$ 
and the other steps are routine. In light of the next (in)equalities

$$
\begin{gathered}
\inf \left(P^{*}\right)=-\operatorname{pd}_{R}(P) \\
\sup \left(P^{*}\right) \leqslant \operatorname{fd}_{R}\left(P^{*}\right)=\operatorname{pd}_{R}\left(P^{*}\right)=-\inf (P)
\end{gathered}
$$

the desired conclusions follow from Theorem 4.1, with $\varphi=\operatorname{id}_{R}: R \rightarrow R$.

Corollary 4.4. Let $P \in \mathcal{D}_{b}^{f}(R)$ be such that $\operatorname{supp}_{R}(P) \supseteq \mathrm{V}(\mathfrak{a}) \bigcap \mathrm{m}-\operatorname{Spec}(R)$ and $\operatorname{pd}_{R}(P)<\infty$. Let $X \in \mathcal{D}(R)$ be such that $\operatorname{co-supp}_{R}(X) \subseteq \mathrm{V}(\mathfrak{a})$ and $K \otimes_{R}^{\mathrm{L}} X \in$ $\mathcal{D}^{f}(R)$.

(a) There are inequalities

$$
\begin{gathered}
\inf \left(\mathbf{R H o m}_{R}(P, X)\right) \leqslant \inf (X)-\inf (P)+n \\
\sup (X)-\operatorname{pd}_{R}(P)-3 n \leqslant \sup \left(\mathbf{R} \operatorname{Hom}_{R}(P, X)\right) \\
\operatorname{amp}(X)-\operatorname{pd}_{R}(P)+\inf (P)-4 n \leqslant \operatorname{amp}\left(\mathbf{R} \operatorname{Hom}_{R}(P, X)\right) .
\end{gathered}
$$

(b) One has $X \simeq 0$ if and only if $\mathbf{R} \operatorname{Hom}_{R}(P, X) \simeq 0$.

(c) For $* \in\{+,-, b\}$, one has $X \in \mathcal{D}_{*}(R)$ if and only if $\mathbf{R H o m}_{R}(P, X) \in \mathcal{D}_{*}(R)$.

Proof. Argue as for Corollary 4.3 , using Theorem 4.2 .

Again, in contrast with the results of Section 3 the point of the next results is that we assume less for $P$ but more for $X$.

Theorem 4.5. Let $P \in \mathcal{D}_{b}(R)$ be a-adically finite such that $\operatorname{pd}_{R}(P)<\infty$ and $\operatorname{supp}_{R}(P) \supseteq \mathrm{V}(\mathfrak{a}) \bigcap \mathrm{m}-\operatorname{Spec}(R)$. Let $X \in \mathcal{D}(R)$ be such that $\operatorname{supp}_{R}(X) \subseteq \mathrm{V}(\mathfrak{a})$. Assume that $K \otimes_{R}^{\mathbf{L}} X \in \mathcal{D}^{f}(R)$.

(a) There are inequalities

$$
\begin{gathered}
\inf \left(\mathbf{R H o m}_{R}(P, X)\right) \leqslant \inf (X)-\inf (P)+3 n \\
\sup (X)-\operatorname{pd}_{R}(P)-2 n \leqslant \sup (\mathbf{R H o m} R(P, X)) \\
\operatorname{amp}(X)+\inf (P)-\operatorname{pd}_{R}(P)-5 n \leqslant \operatorname{amp}(\mathbf{R H o m}(P, X)) .
\end{gathered}
$$

(b) One has $X \simeq 0$ if and only if $\mathbf{R H o m}_{R}(P, X) \simeq 0$.

(c) For $* \in\{+,-, b\}$, one has $X \in \mathcal{D}_{*}(R)$ if and only if $\mathbf{R H o m}_{R}(P, X) \in \mathcal{D}_{*}(R)$.

Proof. We verify (4.5.1) and (4.5.2), using the Hom-evaluation isomorphism

$$
K \otimes_{R}^{\mathbf{L}} \mathbf{R} \operatorname{Hom}_{R}(P, X) \simeq \mathbf{R} \operatorname{Hom}_{R}\left(\mathbf{R} \operatorname{Hom}_{R}(K, P), X\right)
$$

in $\mathcal{D}(R)$; see Fact 2.9. This explains the second step in the next sequence.

$$
\begin{aligned}
\inf \left(\mathbf{R H o m}_{R}(P, X)\right) & \leqslant \inf \left(K \otimes_{R}^{\mathbf{L}} \mathbf{R} \operatorname{Hom}_{R}(P, X)\right) \\
& =\inf \left(\mathbf{R} \operatorname{Hom}_{R}(\mathbf{R H o m} R(K, P), X)\right)
\end{aligned}
$$

The first step is by Fact 2.8(C).

Note that we have $\mathbf{R H o m}_{R}(K, P) \in \mathcal{D}_{\mathrm{b}}^{\mathrm{f}}(R)$, by assumption. Also, we have the self-dual isomorphism

$$
\mathbf{R} \operatorname{Hom}_{R}(K, P) \simeq \Sigma^{-n} K \otimes_{R}^{\mathbf{L}} P
$$

from Fact 2.9 Thus, in the next sequence, the first step is from Fact 2.2

$$
\operatorname{supp}_{R}\left(\mathbf{R H o m}_{R}(K, P)\right)=\operatorname{supp}_{R}(K) \bigcap \operatorname{supp}_{R}(P) \supseteq \mathrm{V}(\mathfrak{a}) \bigcap \mathrm{m}-\operatorname{Spec}(R)
$$

The last step is by assumption, since $\operatorname{supp}_{R}(K)=\mathrm{V}(\mathfrak{a})$. 
Now we compute. The first step in the next sequence is from (4.5.4)

$$
\inf \left(\mathbf{R} \operatorname{Hom}_{R}(K, P)\right)=\inf \left(\Sigma^{-n} K \otimes_{R}^{\mathbf{L}} P\right) \geqslant \inf \left(\Sigma^{-n} K\right)+\inf (P)=-n+\inf (P) .
$$

The second step is from Fact $2.8(\mathrm{C})$, and the third follows from the equality $\inf (K)=$ 0 . This explains the third inequality in the next sequence

$$
\begin{aligned}
\inf \left(\mathbf{R} \operatorname{Hom}_{R}(P, X)\right) & \leqslant \inf \left(\mathbf{R} \operatorname{Hom}_{R}\left(\mathbf{R} \operatorname{Hom}_{R}(K, P), X\right)\right) \\
& \leqslant \inf (X)-\inf \left(\mathbf{R} \operatorname{Hom}_{R}(K, P)\right)+2 n \\
& \leqslant \inf (X)-\inf (P)+3 n .
\end{aligned}
$$

The second inequality here is from Corollary 4.3, and the first one is from the first paragraph of this proof. This establishes (4.5.1).

For (4.5.2), the first step in the next sequence is from (4.5.4).

$$
\operatorname{pd}_{R}\left(\mathbf{R H o m}_{R}(K, P)\right)=\operatorname{pd}_{R}\left(\Sigma^{-n} K \otimes_{R}^{\mathbf{L}} P\right) \leqslant \operatorname{pd}\left(\Sigma^{-n} K\right)+\operatorname{pd}_{R}(P)=\operatorname{pd}_{R}(P)
$$

The second step is from 2, Theorem 4.1(P)], and the third step is from the equality $\operatorname{pd}_{R}\left(\Sigma^{-n} K\right)=0$. This explains the last step in the next sequence.

$$
\begin{aligned}
\sup \left(\mathbf{R H o m}_{R}(P, X)\right) & \geqslant \sup \left(K \otimes_{R}^{\mathbf{L}} \mathbf{R H o m}_{R}(P, X)\right)-n \\
& =\sup \left(\mathbf{R} \operatorname{Hom}_{R}\left(\mathbf{R} \operatorname{Hom}_{R}(K, P), X\right)\right)-n \\
& \geqslant \sup (X)-\operatorname{pd}_{R}(\mathbf{R H o m} R(K, P))-2 n \\
& \geqslant \sup (X)-\operatorname{pd}_{R}(P)-2 n
\end{aligned}
$$

The first step is from Fact 2.8 (c), the second one is is from (4.5.3), and the third one is from Corollary 4.3 ,

Theorem 4.6. Let $P \in \mathcal{D}_{b}(R)$ be a-adically finite such that $\operatorname{pd}_{R}(P)<\infty$ and $\operatorname{supp}_{R}(P) \supseteq \mathrm{V}(\mathfrak{a}) \bigcap \mathrm{m}-\operatorname{Spec}(R)$. Let $X \in \mathcal{D}(R)$ be such that co-supp ${ }_{R}(X) \subseteq \mathrm{V}(\mathfrak{a})$. Assume that $K \otimes{ }_{R} \mathbf{L} X \in \mathcal{D}^{f}(R)$.

(a) There are inequalities

$$
\begin{gathered}
\inf \left(\mathbf{R H o m}_{R}(P, X)\right) \leqslant \inf (X)-\inf (P)+2 n \\
\sup (X)-\operatorname{pd}_{R}(P)-4 n \leqslant \sup \left(\mathbf{R} \operatorname{Hom}_{R}(P, X)\right) \\
\operatorname{amp}(X)+\inf (P)-\operatorname{pd}_{R}(P)-6 n \leqslant \operatorname{amp}(\mathbf{R H o m}(P, X)) .
\end{gathered}
$$

(b) One has $X \simeq 0$ if and only if $\mathbf{R} \operatorname{Hom}_{R}(P, X) \simeq 0$.

(c) For $* \in\{+,-, b\}$, one has $X \in \mathcal{D}_{*}(R)$ if and only if $\mathbf{R H o m}_{R}(P, X) \in \mathcal{D}_{*}(R)$.

Proof. Argue as for Theorem 4.5, using Corollary 4.4 in place of 4.3

\section{Bounding Homology III: Modules}

The next results show how one can replace a faithfulness hypothesis with appropriate support assumptions, beginning with the projective situation.

Proposition 5.1. Let $P$ be a projective $R$-module such that $\mathrm{V}(\mathfrak{a}) \cap \mathrm{m}-\operatorname{Spec}(R) \subseteq$ $\operatorname{supp}_{R}(P)$. Let $Y \in \mathcal{D}(R)$ be such that $\operatorname{supp}_{R}(Y) \subseteq \mathrm{V}(\mathfrak{a})$ or $\operatorname{co-supp}_{R}(Y) \subseteq \mathrm{V}(\mathfrak{a})$.

(a) Then we have $\operatorname{Supp}_{R}(P)=\operatorname{supp}_{R}(P) \supseteq \mathrm{V}(\mathfrak{a})$.

(b) There is a projective $R$-module $Q$ such that $P \oplus Q$ is faithfully projective and $\mathbf{R H o m}_{R}(P \oplus Q, Y) \simeq \mathbf{R H o m}_{R}(P, Y)$. 
(c) One has $\mathrm{H}_{i}\left(\operatorname{RHom}_{R}(P, Y)\right) \neq 0$ if and only if $\mathrm{H}_{i}(Y) \neq 0$, so there are equalities

$$
\begin{aligned}
\inf \left(\mathbf{R H o m}_{R}(P, Y)\right) & =\inf (Y) \\
\sup \left(\mathbf{R H o m}_{R}(P, Y)\right) & =\sup (Y) \\
\operatorname{amp}\left(\mathbf{R H o m}_{R}(P, Y)\right) & =\operatorname{amp}(Y) .
\end{aligned}
$$

(d) One has $Y \simeq 0$ if and only if $\mathbf{R} \operatorname{Hom}_{R}(P, Y) \simeq 0$.

(e) For $* \in\{+,-, b\}$, one has $Y \in \mathcal{D}_{*}(R)$ if and only if $\mathbf{R H o m}_{R}(P, Y) \in \mathcal{D}_{*}(R)$.

Proof. Being projective, the module $P$ is locally free. It follows readily that we have $\operatorname{Supp}_{R}(P)=\operatorname{supp}_{R}(P)$. This also implies that the characteristic function $f: \operatorname{Spec}(R) \rightarrow\{0,1\}$ for $\operatorname{Supp}_{R}(P)$, given by the formula

$$
f(\mathfrak{p}):= \begin{cases}1 & \text { if } P_{\mathfrak{p}} \neq 0 \\ 0 & \text { if } P_{\mathfrak{p}}=0\end{cases}
$$

is locally constant. Thus, there is a decomposition $R \cong R^{\prime} \times R^{\prime \prime}$ such that, under the canonical identification $\operatorname{Spec}(R)=\operatorname{Spec}\left(R^{\prime}\right) \bigsqcup \operatorname{Spec}\left(R^{\prime \prime}\right)$, we have $\operatorname{Spec}\left(R^{\prime}\right)=$ $\operatorname{Supp}_{R}(P)$. From this, it follows that we have $P \cong P^{\prime} \times 0$ for some projective $R^{\prime}$-module $P^{\prime}$ with

$$
\operatorname{Supp}_{R^{\prime}}\left(P^{\prime}\right)=\operatorname{Supp}_{R}(P)=\operatorname{Spec}\left(R^{\prime}\right) .
$$

(We obtain $P^{\prime}$ as the localization of $P$ at the idempotent $e_{2}=(0,1)$.) In particular, $P^{\prime}$ is faithfully projective over $R^{\prime}$.

(国) It remains to show that $\operatorname{Supp}_{R}(P) \supseteq \mathrm{V}(\mathfrak{a})$, so let $\mathfrak{p} \in \mathrm{V}(\mathfrak{a})$. Fix a maximal ideal $\mathfrak{m} \supseteq \mathfrak{p} \supseteq \mathfrak{a}$, so we have

$$
\mathfrak{m} \in \mathrm{V}(\mathfrak{a}) \bigcap \mathrm{m}-\operatorname{Spec}(R) \subseteq \operatorname{supp}_{R}(P)=\operatorname{Supp}_{R}(P)=\operatorname{Spec}\left(R^{\prime}\right) .
$$

The decomposition $\operatorname{Spec}(R)=\operatorname{Spec}\left(R^{\prime}\right) \bigsqcup \operatorname{Spec}\left(R^{\prime \prime}\right)$ says that we have $\mathfrak{m}=\mathfrak{m}^{\prime} \times R^{\prime \prime}$ for some maximal ideal $\mathfrak{m}^{\prime} \in \mathfrak{m}$-Spec $\left(R^{\prime}\right)$. Thus, the containment $\mathfrak{p} \subseteq \mathfrak{m}$ implies that we have $\mathfrak{p}=\mathfrak{p}^{\prime} \times R^{\prime \prime}$ for some prime ideal $\mathfrak{p}^{\prime} \in \operatorname{Spec}\left(R^{\prime}\right)$; in other words, we have $\mathfrak{p} \in \operatorname{Spec}\left(R^{\prime}\right)=\operatorname{Supp}_{R}(P)$, as desired.

(b) We have already seen that $P \cong P^{\prime} \times 0$ for some faithfully projective $R^{\prime}$ module $P^{\prime}$. Set $Q:=0 \times R^{\prime \prime}$. It is straightforward to show that $P \oplus Q \cong P^{\prime} \times R^{\prime \prime}$ is faithfully projective.

Assume for this paragraph that we have co-supp $\operatorname{sun}_{R}(Y) \subseteq \mathrm{V}(\mathfrak{a})$. We prove that this implies that $\operatorname{supp}_{R}(Y) \subseteq \operatorname{supp}_{R}(P)$. The key point here is from [29, Proposition 4.7(b)] which says that $\operatorname{supp}_{R}(Y)$ and $c 0-\operatorname{supp}_{R}(Y)$ have the same maximal elements with respect to containment. Let $\mathfrak{p} \in \operatorname{supp}_{R}(Y)$, and let $\mathfrak{q} \supseteq \mathfrak{p}$ be maximal in $\operatorname{supp}_{R}(Y)$ with respect to containment. It follows that

$$
\mathfrak{q} \in \operatorname{co}_{-\operatorname{supp}_{R}}(Y) \subseteq \mathrm{V}(\mathfrak{a}) \subseteq \operatorname{supp}_{R}(P)=\operatorname{Spec}\left(R^{\prime}\right) .
$$

As in the proof of part (国), the condition $\mathfrak{p} \subseteq \mathfrak{q}$ implies that $\mathfrak{p} \in \operatorname{supp}_{R}(P)$.

In the alternate case $\operatorname{supp}_{R}(Y) \subseteq \mathrm{V}(\mathfrak{a})$, part (国) implies that $\operatorname{supp}_{R}(Y) \subseteq$ $\operatorname{supp}_{R}(P)$. Thus, we assume for the rest of the proof that $\operatorname{supp}_{R}(Y) \subseteq \operatorname{supp}_{R}(P)=$ $\operatorname{Spec}\left(R^{\prime}\right)=\mathrm{V}\left(e_{2}\right)$. It follows that $\operatorname{Supp}_{R}(Y) \subseteq \mathrm{V}\left(e_{2}\right)=\operatorname{Spec}\left(R^{\prime}\right)$ by Fact 2.6 Again localizing at $e_{2}$, we conclude that $Y \simeq Y^{\prime} \times 0$ for some $Y^{\prime} \in \mathcal{D}\left(R^{\prime}\right)$. From 
this we have the following sequence which gives the desired conclusion:

$$
\begin{aligned}
\mathbf{R H o m}_{R}(P \oplus Q, Y) & \simeq \mathbf{R H o m}_{R^{\prime} \times R^{\prime \prime}}\left(P^{\prime} \times R^{\prime \prime}, Y^{\prime} \times 0\right) \\
& \simeq \mathbf{R H o m}_{R^{\prime}}\left(P^{\prime}, Y^{\prime}\right) \times \mathbf{R} \operatorname{Hom}_{R^{\prime \prime}}\left(R^{\prime \prime}, 0\right) \\
& \simeq \mathbf{R H o m}_{R^{\prime}}\left(P^{\prime}, Y^{\prime}\right) \times 0 \\
& \simeq \mathbf{R H o m}_{R^{\prime}}\left(P^{\prime}, Y^{\prime}\right) \times \mathbf{R} \operatorname{Hom}_{R^{\prime \prime}}(0,0) \\
& \simeq \mathbf{R} \operatorname{Hom}_{R^{\prime} \times R^{\prime \prime}}\left(P^{\prime} \times 0, Y^{\prime} \times 0\right) \\
& \simeq \mathbf{R} \operatorname{Hom}_{R}(P, Y) .
\end{aligned}
$$

(ㄷ) - (因) The fact that $P \oplus Q$ is faithfully projective over $R$ implies that $\mathrm{H}_{i}(Y) \neq 0$ if and only if $\mathrm{H}_{i}\left(\mathbf{R H o m}_{R}(P \oplus Q, Y)\right) \neq 0$, that is, if and only if $\mathrm{H}_{i}\left(\mathbf{R H o m}_{R}(P, Y)\right) \neq$ 0 . The desired conclusions now follow directly.

The next two results show how faithful flatness can be relaxed.

Proposition 5.2. Let $F$ be a flat $R$-module, and let $Y \in \mathcal{D}(R)$ be such that we have $\operatorname{Supp}_{R}(Y) \bigcap \mathrm{m}-\operatorname{Spec}(R) \subseteq \operatorname{supp}_{R}(F)$, e.g., such that $\operatorname{supp}_{R}(Y) \subseteq \mathrm{V}(\mathfrak{a})$ and $\mathrm{V}(\mathfrak{a}) \bigcap \mathrm{m}-\operatorname{Spec}(R) \subseteq \operatorname{supp}_{R}(F)$.

(a) One has $\mathrm{H}_{i}\left(F \otimes_{R}^{\mathrm{L}} Y\right) \neq 0$ if and only if $\mathrm{H}_{i}(Y) \neq 0$, so there are equalities

$$
\begin{aligned}
\inf \left(F \otimes_{R}^{\mathbf{L}} Y\right) & =\inf (Y) \\
\sup \left(F \otimes_{R}^{\mathbf{L}} Y\right) & =\sup (Y) \\
\operatorname{amp}\left(F \otimes_{R}^{\mathbf{L}} Y\right) & =\operatorname{amp}(Y) .
\end{aligned}
$$

(b) One has $Y \simeq 0$ if and only if $F \otimes_{R}^{\mathbf{L}} Y \simeq 0$.

(c) For each $* \in\{+,-, b\}$, one has $Y \in \mathcal{D}_{*}(R)$ if and only if $F \otimes_{R}^{\mathbf{L}} Y \in \mathcal{D}_{*}(R)$.

Proof. Note that if $\operatorname{supp}_{R}(Y) \subseteq \mathrm{V}(\mathfrak{a})$, then $\operatorname{Supp}_{R}(Y) \subseteq \mathrm{V}(\mathfrak{a})$ by 29, Proposition 3.15(a)]; if we also have $\mathrm{V}(\mathfrak{a}) \bigcap \mathrm{m}-\operatorname{Spec}(R) \subseteq \operatorname{supp}_{R}(F)$, then it follows that $\operatorname{Supp}_{R}(Y) \bigcap \mathrm{m}-\operatorname{Spec}(R) \subseteq \mathrm{V}(\mathfrak{a}) \bigcap \mathrm{m}-\operatorname{Spec}(R) \subseteq \operatorname{supp}_{R}(F)$.

As in the proof of Proposition [5.1] it suffices to show that for each $i \in \mathbb{Z}$ one has $\mathrm{H}_{i}\left(F \otimes_{R}^{\mathbf{L}} Y\right) \neq 0$ if and only if $\mathrm{H}_{i}(Y) \neq 0$. Since $F$ is a flat $R$-module, we have $\mathrm{H}_{i}\left(F \otimes_{R}^{\mathrm{L}} Y\right) \cong F \otimes_{R} \mathrm{H}_{i}(Y)$, so the forward implication in the previous sentence is routine. For the converse, assume that $\mathrm{H}_{i}(Y) \neq 0$, and let

$$
\mathfrak{m} \in \operatorname{Supp}_{R}\left(\mathrm{H}_{i}(Y)\right) \bigcap \mathrm{m}-\operatorname{Spec}(R) \subseteq \operatorname{Supp}_{R}(Y) \bigcap \mathrm{m}-\operatorname{Spec}(R) \subseteq \operatorname{supp}_{R}(F) .
$$

It follows that the flat $R_{\mathfrak{m}}$-module $F_{\mathfrak{m}}$ has $\mathfrak{m} \in \operatorname{supp}_{R_{\mathfrak{m}}}\left(F_{\mathfrak{m}}\right)$; in other words, $F_{\mathfrak{m}}$ is faithfully flat over $R_{\mathfrak{m}}$. By assumption, we have $\mathrm{H}_{i}(Y)_{\mathfrak{m}} \neq 0$, so

$$
0 \neq F_{\mathfrak{m}} \otimes_{R_{\mathfrak{m}}} \mathrm{H}_{i}(Y)_{\mathfrak{m}} \cong \mathrm{H}_{i}\left(F \otimes_{R} Y\right)_{\mathfrak{m}} .
$$

We conclude that $\mathrm{H}_{i}\left(F \otimes_{R} Y\right) \neq 0$, as desired.

Proposition 5.3. Let $F$ be a flat $R$-module with $\mathrm{V}(\mathfrak{a}) \cap \mathrm{m}-\operatorname{Spec}(R) \subseteq \operatorname{supp}_{R}(F)$. Let $Y \in \mathcal{D}(R)$ be such that $\operatorname{co}^{-\operatorname{supp}_{R}}(Y) \subseteq \mathrm{V}(\mathfrak{a})$.

(a) There are (in) equalities

$$
\begin{gathered}
\inf \left(F \otimes_{R}^{\mathbf{L}} Y\right)=\inf (Y) \\
\sup (Y)-2 n \leqslant \sup \left(F \otimes_{R}^{\mathbf{L}} Y\right) \\
\operatorname{amp}(Y)-2 n \leqslant \operatorname{amp}\left(F \otimes_{R}^{\mathbf{L}} Y\right) .
\end{gathered}
$$


(b) One has $Y \simeq 0$ if and only if $F \otimes_{R}^{\mathrm{L}} Y \simeq 0$.

(c) For each $* \in\{+,-, b\}$, one has $Y \in \mathcal{D}_{*}(R)$ if and only if $F \otimes_{R}^{\mathbf{L}} Y \in \mathcal{D}_{*}(R)$.

Proof. For the infimum computation, we begin with two applications of Fact 2.8(c).

$$
\inf (Y) \leqslant \inf \left(F \otimes_{R}^{\mathbf{L}} Y\right) \leqslant \inf \left(F \otimes_{R}^{\mathbf{L}} Y \otimes_{R}^{\mathbf{L}} K\right)=\inf \left(Y \otimes_{R}^{\mathbf{L}} K\right)=\inf (Y)
$$

The third step here is from Proposition $5.2($ (a) $)$, using the condition $\operatorname{supp}_{R}\left(K \otimes_{R}^{\mathrm{L}} Y\right) \subseteq$ $\mathrm{V}(\mathfrak{a})$. The fourth step is from Fact 2.11(a).

For the supremum bound, we argue similarly

$$
\begin{aligned}
\sup \left(F \otimes_{R}^{\mathrm{L}} Y\right) & \geqslant \sup \left(F \otimes_{R}^{\mathrm{L}} Y \otimes_{R}^{\mathrm{L}} K\right)-n \\
& =\sup \left(Y \otimes_{R}^{\mathrm{L}} K\right)-n \\
& =\sup (Y)-2 n
\end{aligned}
$$

using Fact 2.8(c), Proposition 5.2(国), and Fact 2.11(国).

For perspective in the injective versions of this section, we recall the following. Given an injective $R$-module $I \cong \bigoplus_{\mathfrak{p} \in \operatorname{Spec}(R)} E_{R}(R / \mathfrak{p})^{\left(\mu_{\mathfrak{p}}\right)}$, one has

$$
\begin{aligned}
\operatorname{supp}_{R}(I) & =\left\{\mathfrak{p} \in \operatorname{Spec}(R) \mid \mu_{\mathfrak{p}} \neq \emptyset\right\} \\
\operatorname{co}_{-} \operatorname{Supp}_{R}(I) & =\left\{\mathfrak{q} \in \operatorname{Spec}(R) \mid \text { there is a } \mathfrak{p} \in \operatorname{Spec}(R) \text { such that } \mathfrak{q} \subseteq \mathfrak{p} \text { and } \mu_{\mathfrak{p}} \neq \emptyset\right\}
\end{aligned}
$$

by [29, Propositions 3.8 and 6.3].

Proposition 5.4. Let $I$ be an injective $R$-module with $\mathrm{V}(\mathfrak{a}) \subseteq \operatorname{co}^{-\operatorname{supp}_{R}}(I)$, e.g., with $\mathrm{V}(\mathfrak{a}) \bigcap \mathrm{m}-\operatorname{Spec}(R) \subseteq \operatorname{supp}_{R}(I)$. Let $Y \in \mathcal{D}(R)$ be such that $\operatorname{supp}_{R}(Y) \subseteq \mathrm{V}(\mathfrak{a})$.

(a) One has $\mathrm{H}_{i}\left(\mathbf{R H o m}_{R}(Y, I)\right) \neq 0$ if and only if $\mathrm{H}_{-i}(Y) \neq 0$, so there are equalities

$$
\begin{aligned}
& \inf \left(\mathbf{R H o m}_{R}(Y, I)\right)=-\sup (Y) \\
& \sup \left(\mathbf{R} \operatorname{Hom}_{R}(Y, I)\right)=-\inf (Y) \\
& \operatorname{amp}\left(\mathbf{R H o m}_{R}(Y, I)\right)=\operatorname{amp}(Y) .
\end{aligned}
$$

(b) One has $Y \simeq 0$ if and only if $\mathbf{R} \operatorname{Hom}_{R}(Y, I) \simeq 0$.

(c) For $* \in\{+,-, b\}$, one has $Y \in \mathcal{D}_{*}(R)$ if and only if $\mathbf{R H o m}_{R}(Y, I) \in \mathcal{D}_{*}(R)$.

Proof. Assume first that $\mathrm{V}(\mathfrak{a}) \subseteq \operatorname{co-supp}_{R}(I)$. Again, it suffices to show that for each $i \in \mathbb{Z}$ one has $\mathrm{H}_{i}\left(\mathbf{R H o m}_{R}(Y, I)\right) \neq 0$ if and only if $\mathrm{H}_{-i}(Y) \neq 0$. Since $I$ is an injective $R$-module, we have

$$
\mathrm{H}_{i}\left(\mathbf{R} \operatorname{Hom}_{R}(Y, I)\right) \cong \operatorname{Hom}_{R}\left(\mathrm{H}_{-i}(Y), I\right) \simeq \mathbf{R H o m}_{R}\left(\mathrm{H}_{-i}(Y), I\right)
$$

so the forward implication in the previous sentence is routine. For the converse, assume that $\mathrm{H}_{-i}(Y) \neq 0$, and let $\mathfrak{p} \in \operatorname{supp}_{R}\left(\mathrm{H}_{-i}(Y)\right)$. Note that the condition $\operatorname{supp}_{R}(Y) \subseteq \mathrm{V}(\mathfrak{a})$ implies that we have

$$
\operatorname{supp}_{R}\left(\mathrm{H}_{-i}(Y)\right) \subseteq \operatorname{Supp}_{R}\left(\mathrm{H}_{-i}(Y)\right) \subseteq \operatorname{Supp}_{R}(Y) \subseteq \mathrm{V}(\mathfrak{a})
$$

by Fact 2.6. It follows that

$$
\mathfrak{p} \in \operatorname{supp}_{R}\left(\mathrm{H}_{-i}(Y)\right) \subseteq \mathrm{V}(\mathfrak{a}) \subseteq \operatorname{co-supp}_{R}(I) .
$$

We conclude that $\mathfrak{p} \in \operatorname{supp}_{R}\left(\mathrm{H}_{-i}(Y)\right) \cap \operatorname{co-supp}_{R}(I)$, so $\mathbf{R H o m}_{R}\left(\mathrm{H}_{-i}(Y), I\right) \not \chi 0$ by Fact 2.2 as desired.

Assume next that $\mathrm{V}(\mathfrak{a}) \bigcap \mathrm{m}-\operatorname{Spec}(R) \subseteq \operatorname{supp}_{R}(I)$. We need to show that $\mathrm{V}(\mathfrak{a}) \subseteq$ co-supp $\operatorname{su}_{R}(I)$, so let $\mathfrak{p} \in \mathrm{V}(\mathfrak{a})$. Fix a maximal ideal $\mathfrak{m}$ of $R$ such that $\mathfrak{m} \supseteq \mathfrak{p} \supseteq \mathfrak{a}$, so we have $\mathfrak{m} \in \mathrm{V}(\mathfrak{a}) \bigcap \mathrm{m}$-Spec $(R) \subseteq \operatorname{supp}_{R}(I) \subseteq \operatorname{co-supp}_{R}(I)$ by the notes preceding 
the statement of this result. Since we have $\mathfrak{p} \subseteq \mathfrak{m} \in \operatorname{co}-\operatorname{supp}_{R}(I)$, these notes also imply that $\mathfrak{p} \in \mathrm{co-supp}_{R}(I)$, as desired.

Proposition 5.5. Let $I$ be an injective $R$-module with $\mathrm{V}(\mathfrak{a}) \subseteq \operatorname{co-supp}_{R}(I)$, e.g., with $\mathrm{V}(\mathfrak{a}) \bigcap \mathrm{m}-\operatorname{Spec}(R) \subseteq \operatorname{supp}_{R}(I)$. Let $Y \in \mathcal{D}(R)$ be with $\operatorname{co}^{-\operatorname{supp}_{R}}(Y) \subseteq \mathrm{V}(\mathfrak{a})$.

(a) There are (in) equalities

$$
\begin{gathered}
\inf \left(\mathbf{R H o m}_{R}(Y, I)\right) \leqslant 2 n-\sup (Y) \\
\sup \left(\mathbf{R} \operatorname{Hom}_{R}(Y, I)\right)=-\inf (Y) \\
\operatorname{amp}(Y)-2 n \leqslant \operatorname{amp}\left(\mathbf{R H o m}{ }_{R}(Y, I)\right) .
\end{gathered}
$$

(b) One has $Y \simeq 0$ if and only if $\mathbf{R H o m}_{R}(Y, I) \simeq 0$.

(c) For $* \in\{+,-, b\}$, one has $Y \in \mathcal{D}_{*}(R)$ if and only if $\mathbf{R H o m}_{R}(Y, I) \in \mathcal{D}_{*}(R)$.

Proof. We argue as for Proposition 5.3, using Proposition 5.4. In the next sequence, the first and last steps are from Fact 2.8(C).

$$
\begin{aligned}
\inf \left(\mathbf{R H o m}_{R}(Y, I)\right) & \leqslant \inf \left(K \otimes_{R}^{\mathbf{L}} \mathbf{R H o m}_{R}(Y, I)\right) \\
& =\inf \left(\mathbf{R} \operatorname{Hom}_{R}\left(\mathbf{R} \operatorname{Hom}_{R}(K, Y), I\right)\right) \\
& =-\sup (\mathbf{R H o m} R(K, Y)) \\
& =-\sup \left(\Sigma^{-n} K \otimes_{R}^{\mathbf{L}} Y\right) \\
& =n-\sup \left(K \otimes_{R}^{\mathbf{L}} Y\right) \\
& \leqslant 2 n-\sup (Y)
\end{aligned}
$$

The second step here is by Hom-evaluation 2.9. The third step is by Proposition 5.4 since the fact that $\mathfrak{a}$ annihilates $\mathrm{H}\left(\mathbf{R} \operatorname{Hom}_{R}(K, Y)\right)$ implies that we have $\operatorname{supp}_{R}\left(\mathbf{R} \operatorname{Hom}_{R}(K, Y)\right) \subseteq \mathrm{V}(\mathfrak{a})$. The fourth step is by the self-dual nature 2.9 of $K$, and the fifth step is routine. This establishes (5.5.1).

For (5.5.2), we being the next sequence with two applications of Fact 2.8 (c).

$$
\begin{aligned}
-\inf (Y) & \geqslant \sup \left(\mathbf{R H o m}_{R}(Y, I)\right) \\
& \geqslant \sup \left(K \otimes_{R}^{\mathbf{L}} \mathbf{R} \operatorname{Hom}_{R}(Y, I)\right)-n \\
& =\sup \left(\mathbf{R} \operatorname{Hom}_{R}\left(\mathbf{R H o m}_{R}(K, Y), I\right)\right)-n \\
& =-\inf \left(\mathbf{R H o m}_{R}(K, Y)\right)-n \\
& =-\inf \left(\Sigma^{-n} K \otimes_{R}^{\mathbf{L}} Y\right)-n \\
& =-\inf \left(K \otimes_{R}^{\mathbf{L}} Y\right) \\
& =-\inf (Y)
\end{aligned}
$$

The remaining steps are by Hom-evaluation 2.9 Proposition 5.4 the self-dual nature 2.9 of $K$, a routine computation, and Fact 2.11(a).

\section{Applications}

We end with an indication of some of the applications of our boundedness results, following Foxby and Iyengar [10]. See also [25] for other applications.

Theorem 6.1. Let $\left(Q, \mathfrak{m}_{Q}, k\right) \rightarrow\left(R, \mathfrak{m}_{R}\right) \stackrel{\varphi}{\rightarrow}\left(S, \mathfrak{m}_{S}\right)$ be local ring homomorphisms such that $\mathfrak{a} \subseteq \operatorname{rad}\left(\mathfrak{m}_{Q} R\right)$. Assume that $F \in \mathcal{D}_{b}(S)$ is $\mathfrak{a} S$-adically finite over $S$ with $F \nsucceq 0$ and $\operatorname{fd}_{R}(F)<\infty$. Then there are inequalities

$$
\mathrm{fd}_{Q}(R)+\inf (F)-n \leqslant \mathrm{fd}_{Q}(F) \leqslant \mathrm{fd}_{Q}(R)+\mathrm{fd}_{R}(F) .
$$


In particular, the quantities $\operatorname{fd}_{Q}(R)$ and $\mathrm{fd}_{Q}(F)$ are simultaneously finite.

Proof. Note that the fact that the map $R \rightarrow S$ is local implies that $\mathfrak{a} S \neq S$.

The second of our desired inequalities is from [2, Corollary $4.2(\mathrm{bF})]$. To verify the first of our desired inequalities, we argue as in [10, Theorem 3.2], using Theorem 4.1 in place of [10, Theorem 3.1]. The first step in the next display is from Fact 2.8(c).

$$
\begin{aligned}
\operatorname{fd}_{Q}(F) & \geqslant \sup \left(k \otimes_{Q}^{\mathbf{L}} F\right) \\
& =\sup \left(\left(k \otimes_{Q}^{\mathbf{L}} R\right) \otimes_{R}^{\mathbf{L}} F\right) \\
& \geqslant \sup \left(k \otimes_{Q}^{\mathbf{L}} R\right)+\inf (F)-n \\
& =\operatorname{fd}_{Q}(R)+\inf (F)-n
\end{aligned}
$$

The second step is from tensor cancellation $k \otimes_{Q}^{\mathbf{L}} F \simeq\left(k \otimes_{Q}^{\mathbf{L}} R\right) \otimes_{R}^{\mathbf{L}} F$, and the fourth step is [2, Proposition 5.5(F)]. The third step is from Theorem 4.1(国) with $X:=k \otimes_{Q}^{\mathrm{L}} R \in \mathcal{D}_{+}^{\mathrm{f}}(R)$; we need to check that the hypotheses of this result are satisfied. The condition $F \not 00$ implies that $\mathfrak{m}_{S} \in \operatorname{Supp}_{S}(F)=\operatorname{supp}_{S}(F)$, by Fact 2.6. In particular, we have $\mathfrak{m}_{R}=\varphi^{-1}\left(\mathfrak{m}_{S}\right)$, and so $\mathrm{V}(\mathfrak{a}) \cap \mathrm{m}-\operatorname{Spec}(R)=$ $\left\{\mathfrak{m}_{R}\right\} \subseteq \varphi^{*}\left(\operatorname{supp}_{S}(F)\right)$. The homology of $k \otimes_{Q}^{\mathbf{L}} R$ is annihilated by $\mathfrak{m}_{Q} R$, hence the first containment in the next sequence.

$$
\operatorname{supp}_{R}\left(k \otimes_{Q}^{\mathbf{L}} R\right) \subseteq \mathrm{V}\left(\mathfrak{m}_{Q} R\right) \subseteq \mathrm{V}(\mathfrak{a})
$$

The second containment is from the assumption $\mathfrak{a} \subseteq \operatorname{rad}\left(\mathfrak{m}_{Q} R\right)$. Thus, Theorem 4.1(国) applies, as desired.

We conclude with Theorem 1.3 from the introduction. Our argument is based in spirit on that of [10, Theorem 3.3].

Theorem 6.2. Let $(R, \mathfrak{m})$ be a local ring of prime characteristic, and let $\varphi: R \rightarrow R$ be the Frobenius endomorphism. Then the following conditions are equivalent.

(i) $R$ is regular.

(ii) $\varphi^{t}$ is flat for each (equivalently, some) integer $t \geqslant 1$.

(iii) One has $\operatorname{fd}_{R}\left(\varphi^{t} \mathbf{R} \Gamma_{\mathfrak{b}}(R)\right)<\infty$ for some integer $t \geqslant 1$ and some ideal $\mathfrak{b} \subseteq \mathfrak{m}$.

(iv) One has $\operatorname{fd}_{R}\left(\varphi^{t} F\right)<\infty$ for some integer $t \geqslant 1$ for some $\mathfrak{b}$-adically finite $R$-complex $F \not 0$ with $\mathrm{fd}_{R}(F)<\infty$ for some ideal $\mathfrak{b} \subseteq \mathfrak{m}$.

Proof. The equivalence of conditions (ii) and (iii) are from [18, (2.1)]. The implication (iii) $\Longrightarrow$ (iii) is from [2, Corollary $4.2(\mathrm{bF})$ ], since $\mathbf{R} \Gamma_{\mathfrak{b}}(R)$ has finite flat dimension (via the Cech complex). The implication (iii) $\Longrightarrow$ (iv) is from the fact that $\mathbf{R} \Gamma_{\mathfrak{b}}(R)$ has finite flat dimension and is $\mathfrak{b}$-adically finite by [29, Theorem 7.10].

(iv) $\Longrightarrow$ (iii) Assume that $\mathrm{fd}_{R}\left(\varphi^{t} F\right)<\infty$ for some integer $t \geqslant 1$ for some $\mathfrak{b}$ adically finite $R$-complex $F \not 0$ with $\mathrm{fd}_{R}(F)<\infty$ for some ideal $\mathfrak{b} \subseteq \mathfrak{m}$. To prove that $\varphi^{t}$ is flat, it suffices by the proof of [10, Theorem 3.3] to show that $\operatorname{fd}_{R}\left(\varphi^{t} R\right)<\infty$.

Since $\varphi$ is the Frobenius endomorphism, we have $\operatorname{rad}\left(\varphi^{t}(\mathfrak{m}) R\right)=\mathfrak{m} \supseteq \mathfrak{b}$. Thus, the hypotheses of Theorem 6.1 are satisfied with the homomorphisms $R \stackrel{\varphi^{t}}{\longrightarrow} R \stackrel{\mathrm{id}}{\longrightarrow} R$ and $\mathfrak{a}=\mathfrak{b}$. We conclude that $\operatorname{fd}_{R}\left(\varphi^{t} R\right) \leqslant \operatorname{fd}_{R}\left(\varphi^{t} F\right)-\inf (F)+n<\infty$ as desired.

\section{ACKNOWLEDGMENTs}

We are grateful to Srikanth Iyengar, Liran Shaul, and Amnon Yekutieli for helpful comments about this work. 


\section{REFERENCES}

1. L. Alonso Tarrío, A. Jeremías López, and J. Lipman, Local homology and cohomology on schemes, Ann. Sci. École Norm. Sup. (4) 30 (1997), no. 1, 1-39. MR 1422312 (98d:14028)

2. L. L. Avramov and H.-B. Foxby, Homological dimensions of unbounded complexes, J. Pure Appl. Algebra 71 (1991), 129-155. MR 93g:18017

3. L. L. Avramov, H.-B. Foxby, and S. Halperin, Differential graded homological algebra, in preparation.

4. D. Benson, S. B. Iyengar, and H. Krause, Local cohomology and support for triangulated categories, Ann. Sci. Éc. Norm. Supér. (4) 41 (2008), no. 4, 573-619. MR 2489634 (2009k:18012)

5. Colocalizing subcategories and cosupport, J. Reine Angew. Math. 673 (2012), 161207. MR 2999131

6. L. W. Christensen, H.-B. Foxby, and A. Frankild, Restricted homological dimensions and Cohen-Macaulayness, J. Algebra 251 (2002), no. 1, 479-502. MR 1900297 (2003e:13022)

7. L. W. Christensen and H. Holm, Ascent properties of Auslander categories, Canad. J. Math. 61 (2009), no. 1, 76-108. MR 2488450

8. H.-B. Foxby, Isomorphisms between complexes with applications to the homological theory of modules, Math. Scand. 40 (1977), no. 1, 5-19. MR 0447269 (56 \#5584)

9. - Bounded complexes of flat modules, J. Pure Appl. Algebra 15 (1979), no. 2, 149-172. MR 535182 (83c:13008)

10. H.-B. Foxby and S. Iyengar, Depth and amplitude for unbounded complexes, Commutative algebra. Interactions with Algebraic Geometry, Contemp. Math., vol. 331, Amer. Math. Soc., Providence, RI, 2003, pp. 119-137. MR 2013162

11. A. Frankild and S. Sather-Wagstaff, Reflexivity and ring homomorphisms of finite flat dimension, Comm. Algebra 35 (2007), no. 2, 461-500. MR 2294611

12. R. Hartshorne, Residues and duality, Lecture Notes in Mathematics, No. 20, Springer-Verlag, Berlin, 1966. MR 36 \#5145

13. L L L L L cohomology, A seminar given by A. Grothendieck, Harvard University, Fall, vol. 1961, Springer-Verlag, Berlin, 1967. MR 0224620 (37 \#219)

14. _ Affine duality and cofiniteness, Invent. Math. 9 (1969/1970), 145-164. MR 0257096 (41 \#1750)

15. B. Iversen, Amplitude inequalities for complexes, Ann. Sci. École Norm. Sup. (4) 10 (1977), no. 4, 547-558. MR $58 \# 27966$

16. K.-i. Kawasaki, On a category of cofinite modules which is Abelian, Math. Z. 269 (2011), no. 1-2, 587-608. MR 2836085 (2012h:13026)

17. On a characterization of cofinite complexes. Addendum to "On a category of cofinite modules which is Abelian", Math. Z. 275 (2013), no. 1-2, 641-646. MR 3101824

18. E. Kunz, Characterizations of regular local rings of characteristic p, Amer. J. Math. 91 (1969), 772-784. MR 0252389 (40 \#5609)

19. J. Lipman, Lectures on local cohomology and duality, Local cohomology and its applications (Guanajuato, 1999), Lecture Notes in Pure and Appl. Math., vol. 226, Dekker, New York, 2002, pp. 39-89. MR 1888195 (2003b:13027)

20. E. Matlis, The Koszul complex and duality, Comm. Algebra 1 (1974), 87-144. MR 0344241 (49 \#8980)

21. (80a:13013)

22. Leif Melkersson, Modules cofinite with respect to an ideal, J. Algebra 285 (2005), no. 2, 649-668. MR 2125457 (2006i:13033)

23. M. Porta, L. Shaul, and A. Yekutieli, On the homology of completion and torsion, Algebr. Represent. Theory 17 (2014), no. 1, 31-67. MR 3160712

24. $\longrightarrow$, Cohomologically cofinite complexes, Comm. Algebra 43 (2015), no. 2, 597-615. MR 3274024

25. S. Sather-Wagstaff and R. Wicklein, Adic Foxby classes, preprint (2016), arxiv:1602.03227.

26. - Adic semidualizing complexes, preprint (2015), arxiv:1506.07052.

27. Adically finite chain complexes, preprint (2016), arxiv:1602.03224.

28. Extended local cohomology and local homology, preprint (2016), arxiv:1602.03226.

29. - Support and adic finiteness for complexes, Comm. Algebra, to appear, arXiv: 1401.6925 
30. A.-M. Simon, Some homological properties of complete modules, Math. Proc. Cambridge Philos. Soc. 108 (1990), no. 2, 231-246. MR 1074711 (91k:13008)

31. J.-L. Verdier, Catégories dérivées, SGA $4 \frac{1}{2}$, Springer-Verlag, Berlin, 1977, Lecture Notes in Mathematics, Vol. 569, pp. 262-311. MR 57 \#3132

32. - Des catégories dérivées des catégories abéliennes, Astérisque (1996), no. 239, xii+253 pp. (1997), With a preface by Luc Illusie, Edited and with a note by Georges Maltsiniotis. MR 98c:18007

Department of Mathematical Sciences, Clemson University, O-110 Martin Hall, Box 340975, Clemson, S.C. 29634 USA

E-mail address: ssather@clemson.edu

$U R L:$ https://ssather.people.clemson.edu/

Richard Wicklein, Mathematics and Physics Department, Macmurray College, 447

East College Ave., Jacksonville, IL 62650, USA

E-mail address: richard.wicklein@mac.edu 